\title{
In-Flight Testing of MEMS Pressure Sensors for Flight Loads Determination
}

\author{
Christian Raab*, \\ DLR - Institute of Flight Systems, Braunschweig, 38108, Germany \\ Kai Rohde-Brandenburger ${ }^{\dagger}$ \\ DLR - Institute of Aerodynamics and Flow Technology, Braunschweig, 38108, Germany.
}

The determination and monitoring of structural loads during flight is an important part of every certification program. It must be proved to the certification authorities that the loads occurring during specific flight maneuvers do not exceed the permissible component loads. Usually conventional strain gauges are used to determine structural loads, however an elaborate calibration process is necessary to determine these loads from the measured strains. Recent advances in sensor technology allow determining aerodynamic loads directly from pressure distributions measured by MEMS based sensors. When compared to strain gauges this measurement method has several advantages over conventional strain gauges in terms of installation and calibration costs. Flight tests were carried out with the DLR research glider aircraft Discus-2c in order to compare and investigate the loads determined with the two measuring methods. For this purpose a wing glove equipped with 64 MEMS pressure sensors on the airfoil surface was constructed. Different maneuvers with varying loads were performed during the test flights. A first evaluation of steady flight maneuvers shows that the loads determined from the measured pressure distribution are in very good accordance with the ones determined from the strain gauge measurements. This paper gives an overview of the flight test setup and the data analysis process, results from evaluations of trimmed wings-level flight and steady turn maneuvers are presented and discussed.

\section{Nomenclature}

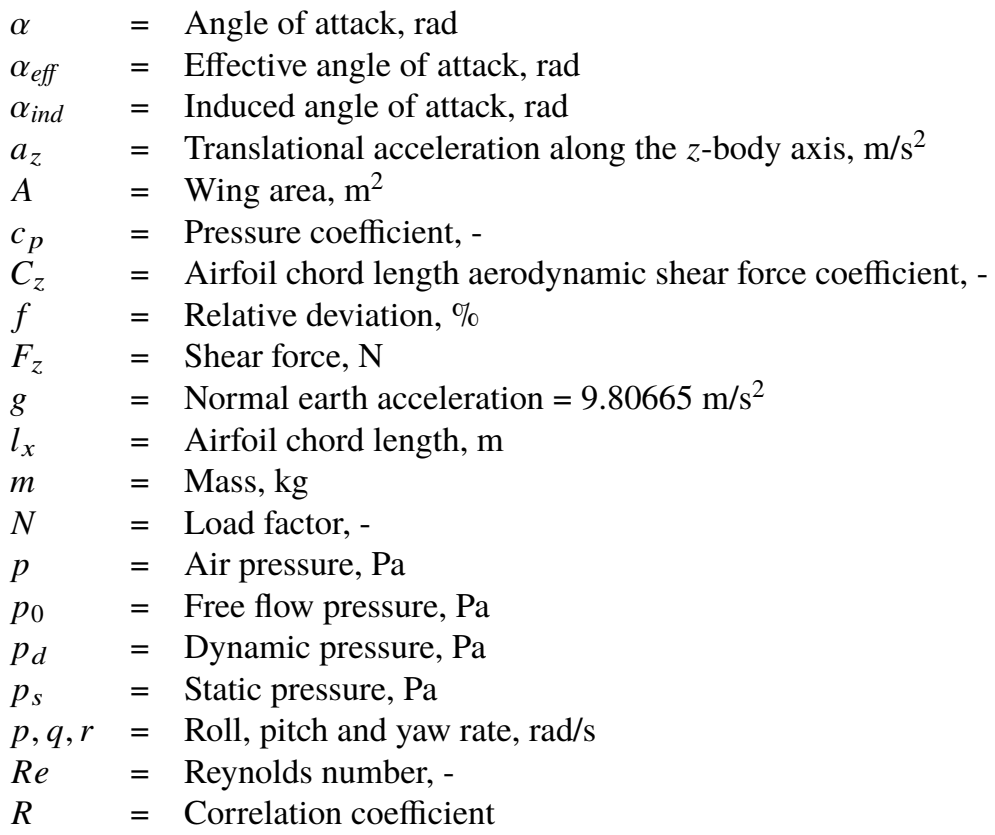

*Research scientist, DLR - Institute of Flight Systems, Lilienthalplatz 7, 38108 Braunschweig, Germany.

${ }^{\dagger}$ Research scientist, DLR - Institute of Aerodynamics and Flow Technology, Braunschweig, Lilienthalplatz 7, 38108 Braunschweig, Germany. 


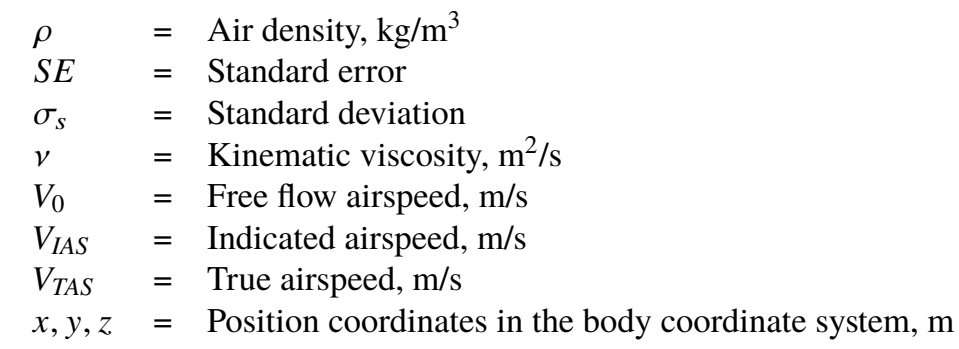

\section{Subscripts}

$\begin{array}{ll}\text { avg } & =\text { Averaged value } \\ C G & =\text { Center of gravity } \\ \text { IMU } & =\text { Inertial Reference Unit } \\ \text { LH,RH } & =\text { Left hand, right hand } \\ \text { lo } & =\text { Lower airfoil surface } \\ m & =\text { Measured value } \\ \text { MEMS } & =\text { MEMS sensor } \\ \text { NB } & =\text { Nose boom } \\ \text { local } & =\text { Local measurement } \\ \text { cor } & =\text { Corrected measurement } \\ \text { offset } & =\text { Offset value } \\ \text { opt } & =\text { Optimized parameter } \\ \text { ref } & =\text { Reference value } \\ \text { SG } & =\text { Strain gauge } \\ \text { total } & =\text { Total value } \\ \text { up } & =\text { Upper airfoil surface } \\ W 1 & =\text { Wing load station No. 1 } \\ x_{c} & =\text { Position along the normalized airfoil chord length } \\ x, y, z & =\text { Axes in the geometric reference coordinate system }\end{array}$

\section{Introduction}

$\mathrm{T}$ HE exact determination of aerodynamic loads acting on the structure is essential for the certification of a new aircraft type. Based on model simulations, the aircraft wing, fuselage and tail components are designed such that they withstand the forces and moments occurring during maneuvers in the required flight envelope. By performing flight tests, it must be proved to the certification authorities that forces and moments working on the structural components during specified maneuvers do not exceed the design limits. The loads measured during the flight test are also used to validate and correct simulation models. These models play an important role for the initial certification, but also later during the operational life of the aircraft: Load models created during the certification process may also be used for health and usage monitoring of each individual aircraft in the fleet.

\section{A. Current State of the Art}

Load measurements of sufficient accuracy and reliability are one of the main prerequisites for the development of a high-quality load model. For this task conventional strain gauges (SG) have been used for a long time [1, 2]. Typically four individual strain gauges are connected to a full-bridge configuration. That way a compensation for temperature changes is possible and arrangements specifically designed for one load case are available. In order to determine the local structural shear force, bending and torsion moment from the measured strains, an elaborate calibration procedure is necessary [3, 4]. The aircraft structure is divided into separate load sections. Each section is usually instrumented with several SG arrangements. For the calibration of the SG system, specific known loads are introduced to the aircraft structure with hydraulic plungers at different positions. Variation of the applied loads, leads to a set of regression equations with which the shear force, bending and torsion moment on each load section can be determined from the measured strains. As the effort for attaching the SGs on the structure and their elec- 
trical wiring is quite high and prone to errors, several other methods have been investigated for measuring structural loads.

Optical fibers have been investigated as an alternative for electrical SGs [5, 6]. By using Fiber-Bragg-Grating (FBG) technology, it is possible to integrate hundreds of strain sensors in a single fiber, thus reducing the effort for wiring. FBGs may also be integrated directly into the aircraft structure and do not cause electromagnetic interference. However, careful calibration is still necessary to determine the actual loads from the strains.

Another approach for the determination of aircraft loads is the direct measurement of aerodynamic pressures acting on the structure. By knowing the pressure distribution around a wing section, forces and moments can be calculated by integration of the local pressure measurements along the wing airfoil. As this approach is close to the physical process causing the structural forces, there is less need for a costly calibration like for the strain based methods. The measurement of pressure distributions on an aircraft structure is of course not only of interest for the determination of loads, but first and foremost of interest for the investigation and validation of the aerodynamic performance. Traditionally, a large number of pressure transducers connected by tubes to pressure taps on the structural surface are used to measure pressure distributions. So-called pressure belts are constructed with this system, measuring the distribution along a wing airfoil. However, this conventional method with pressure tubes has many disadvantages:

- Holes must be drilled into the structure or a smooth fairing must be established on the wing surface without affecting the flow field.

- Storage space and routing for tubes and the pressure transducers cause a high effort for installation.

- The long tubes induce a viscous lag reducing the dynamic range of the measurement system.

- The viscous lag has to be determined for each tube in a calibration process for synchronized pressure measurements.

The pressure tubing method is widely used for wind tunnel experiments because dedicated space can be designed into the scaled models and the pressure transducers can be placed outside the model. Research on high-lift aerodynamics raised the need for measuring pressure distributions in-flight, because the complex high-lift flow is sensitive to Mach and Reynolds numbers and cannot be easily extrapolated from wind tunnel and CFD results [7- -9$]$. Constraints on the tubing length and the limited installation space make the construction of pressure belts for in-flight measurements very expensive and increase the down-time of the aircraft.

Pressure sensors based on Micro-Electro-Mechanical-Systems (MEMS) have features that can overcome the disadvantages mentioned above [10, 11]:

- The small dimensions of only a few millimeters minimizes the installation space and flow disturbances.

- There is no tubing and no viscous lag because the local absolute pressure is measured directly by the sensor.

- Sensors can be integrated in large numbers on a flexible circuit layer, resulting in large local resolution of the measured pressure distribution.

- Each sensor measures also the local temperature which is used for error compensation, resulting in repeatable more accurate sensor measurements.

The significant reduction in installation costs and aircraft down-time made the MEMS pressure sensor technology interesting for the instrumentation of flight tests for certification. In 2001 Boeing and the sensor company Endevco developed a pressure belt system which flew on Boeing 757-300, 737-BBJ, 767-400 and F-18E aircraft [12, 13]. The system consisted of several chip modules which were equipped with a MEMS pressure sensor and a controller circuit for data acquisition. It was successfully applied during flight testing of the Boeing 787 aircraft for a flight load survey [14]. Airbus developed MEMS sensors for measuring the aerodynamic pressure distribution during the flight test of the A350 aircraft. This technology was applied successfully again during the flight test of the A330-neo aircraft and lead to "less data analysis and fewer flights" [15].

\section{B. Focus of Study}

Although the flight test domain has benefited from the use of MEMS pressure sensors, a direct comparison between the strain-based and the pressure-based method for the in-flight determination of flight loads can only rarely be found in the literature. The DLR Institute of Flight Systems was involved in many Airbus certification flight test programs with its expertise in flight data processing and system identification. During this cooperation the idea for an investigation of 
the two methods for the in-flight determination of flight loads was born. In this paper we demonstrate the application of MEMS pressure sensors for flight load determination and compare these loads to the results from SG measurements. For this investigation we performed flight tests with the DLR research aircraft Discus-2c shown in Fig. 1 . This high performance glider airplane is equipped with a system of calibrated SGs for measuring loads on the wing, the fuselage and the horizontal tail. The DLR Institute of Aerodynamics and Flow Technology provided its expertise on sensors for measuring pressure distributions on aircraft structures. Commercial of the shelf MEMS pressure sensors were integrated on flexible circuit strips. These strips were used to construct a wing glove with a total of 64 pressure sensors on the airfoil top and the bottom.

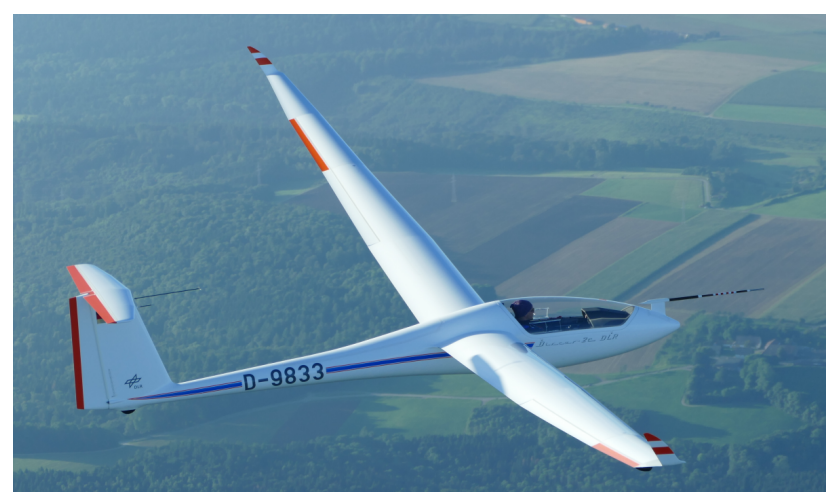

Fig. 1 The DLR Discus-2c research glider.

A flight test program with maneuvers imposing varying loads was performed. During the flight maneuvers the measurements from the SGs and the MEMS pressure sensors were recorded and analyzed after the flights. To the best of the authors knowledge this is the first time that inflight pressure distribution measurements with MEMS sensors around the whole wing airfoil were performed and directly compared to SG measurements.

The paper is organized as follows: In Chapter III the test aircraft, its instrumentation and the performed flight test program are described. It contains as well details about the used MEMS sensors and the construction of the wing glove being used for the measurement of pressure distribution around the wing airfoil section. After the flight test the recorded data were post-processed and corrected. Shear forces for a dedicated wing section were determined from SG and the local pressure measurements. This data analysis process is explained in Chapter IV Shear forces determined from SG and MEMS measurements during different steady flight maneuvers were compared to each other, the results are presented and discussed in Chapter $\mathrm{V}$. Final conclusions and an outlook on future research activities are given in Chapter VI

\section{Experimental Setup}

\section{A. Test Aircraft and Instrumentation}

The Discus-2c is a modern $18 \mathrm{~m}$ class glider without flaps, built in carbon fiber composite by the German manufacturer SchemppHirth (see Fig. 11. It is used as a reference aircraft for performance measurements of new glider design prototypes. For this mission the aerodynamic lift and drag polar have been determined with great accuracy. Performance measurements with the Discus-2c are conducted at regular intervals during the summer meetings of the academic flying club umbrella organization Idaflieg. It was used in an extensive research program for aircraft system identification [16]. The glider has a wing span of $18 \mathrm{~m}$ and an empty weight of $280 \mathrm{~kg}$. Further technical details and envelope limits can be found in Table 1 .

For the flight test campaign the Discus-2c airplane was equipped with several sensors depicted in Fig. 2 Pressure transducers connected to a 5-hole-probe located in a nose boom at the front of the aircraft nose measured the static and the total air pressure as well as the angle of attack (AoA) and the angle of sideslip (AoS). The total air temperature (TAT) was measured with a probe situated at the RH side of the cockpit cover. An inertial reference unit (IMU) located in the equipment bay of the glider measured the accelerations along and the rotation rates around the body axes. The IMU was connected
Table 1 Technical data for the glider aircraft Discus-2c.

\begin{tabular}{|l|r|}
\hline Wing span & $18.00 \mathrm{~m}$ \\
\hline Wing area & $11.39 \mathrm{~m}^{2}$ \\
\hline Aspect ratio & 28.50 \\
\hline Mean aero. chord & $0.685 \mathrm{~m}$ \\
\hline Empty weight & $280 \mathrm{~kg}$ \\
\hline Gross weight & $455 \mathrm{~kg}$ \\
\hline Maneuvering speed $\mathrm{V}_{\mathrm{A}}$ & $190 \mathrm{~km} / \mathrm{h}$ \\
\hline $\begin{array}{l}\text { Permitted load factor } \\
\text { limits at V }\end{array}$ & $-2.65 /+5.30 \mathrm{~g}$ \\
\hline Max. Speed $\mathrm{V}_{\mathrm{NE}}$ & $280 \mathrm{~km} / \mathrm{h}$ \\
\hline Stall Speed & $75 \mathrm{~km} / \mathrm{h}$ \\
\hline $\begin{array}{l}\text { Wing glove airfoil } \\
\text { chord length } l_{c}\end{array}$ & $0.902 \mathrm{~m}$ \\
\hline Wing part area $A_{r e f, W l}$ & $5.141 \mathrm{~m}{ }^{2}$ \\
\hline
\end{tabular}




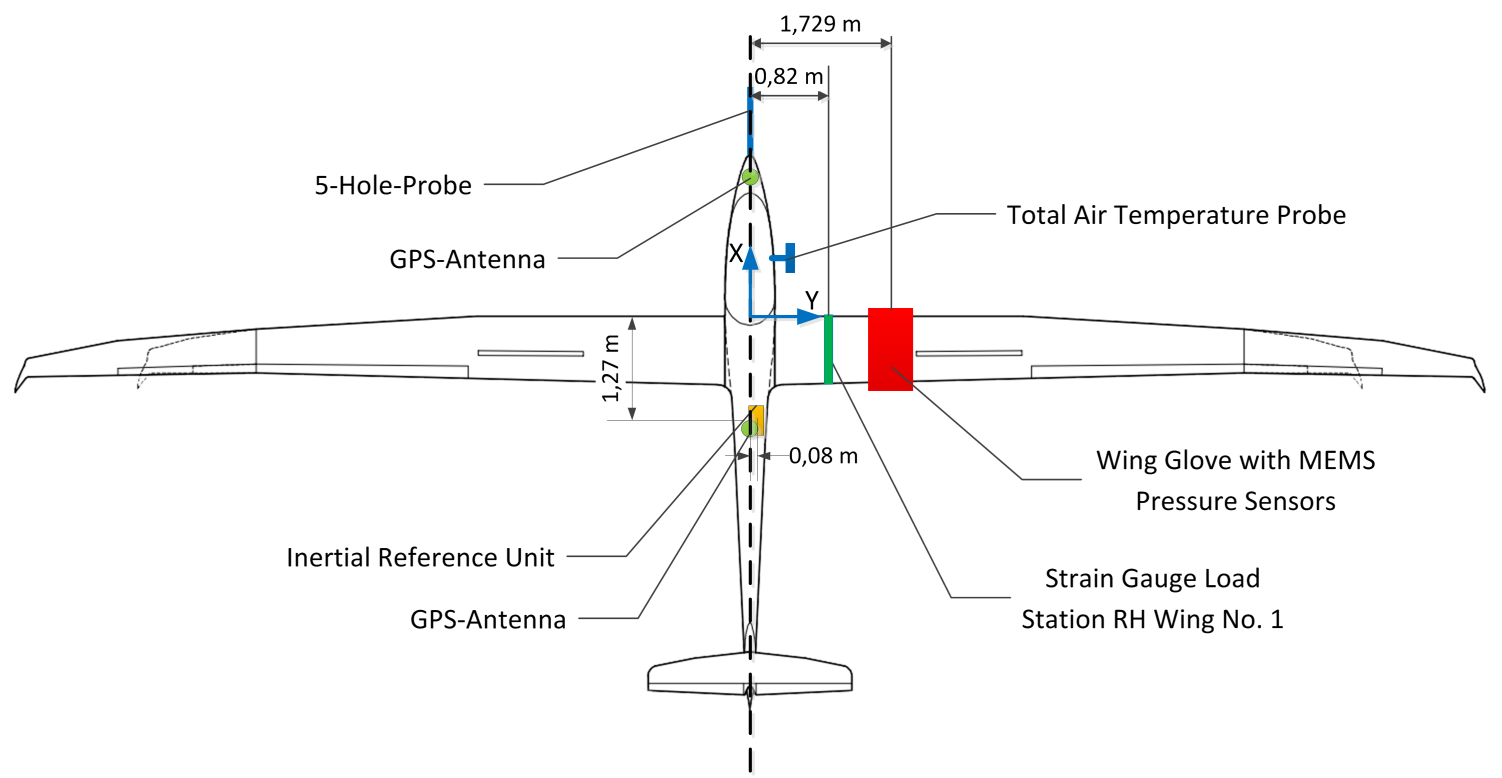

Fig. 2 Flight test sensors on the Discus-2c.

to two GPS antennas which allowed an exact determination of the true heading and a stabilization of the inertial measurements with position updates from the GPS receiver. Besides the geodetic aircraft position, the GPS receiver delivered a common time and data signal for the sensor measurements. In order to record the pilot inputs during the maneuvers, laser distance sensors installed on the steering rods measured the position of the control surfaces such as aileron, elevator and rudder. The deployment of the airbrakes was tracked with additional electrical potentiometers.

During the construction of the Discus-2c a total of 46 four-active-arm SGs were installed inside the aircraft structure. A total of 28 SGs with a load sensitivity for bending moment and shear force were installed in the aircraft wing at dedicated points along the wing span. In the rear fuselage and the horizontal tail 18 SGs were installed with a load sensitivity for shear force and bending moment. The calibration of the SG system followed the traditional procedure [17]: Defined weights in form of sand bags were placed at different positions on the aircraft wing, tail and fuselage structure. For each load case the voltage output of each SG was measured and converted to strain. A regression analysis was performed with the acquired measurements leading to a set of equations, documented in report [17], for each load station on the aircraft structure. With these equations the shear force, bending and torsion moment at each load station can be calculated from the measured strains. The whole calibration campaign lasted nearly 3 weeks with the airplane jacked up in the hangar. Shear force, bending moment and torsion can be calculated from the SG measurements for three load sections at each wing side.

For the flight test only the RH wing was equipped with the wing glove containing the MEMS pressure sensors. Therefore only the RH wing load station No. 1 as depicted in Fig. 2] was considered for the investigation of shear forces determined from the SG and the MEMS pressure measurements. The load equations determined during the calibration process were validated with check loads which were independent from the load cases used for the calibration. A maximum relative error of nearly $\pm 3.6 \%$ was determined for the shear force measurement at the RH wing load station No. 1 with this validation procedure [18].

The measurements from the installed sensor systems, as well as the pressure data from wing glove sensors were recorded by a data acquisition unit stored in the equipment bay of the Discus-2c. All sensor signals were sampled at a rate of $100 \mathrm{~Hz}$ and were processed to a MATLAB file. The data acquisition could be controlled and monitored by the aircraft pilot using a hand-held device. He was also able to set counting markers so that maneuvers could be found more easily in the time history data of the flight. 


\section{B. MEMS Pressure Sensor}

The MEMS-pressure sensors used in this test were BOSCH BMP280 with a footprint of $2 \mathrm{~mm} \times 2.5 \mathrm{~mm}$ shown in Fig. 3. The sensors can measure temperature and pressure directly inside the sensor housing and the raw values can be read out. The factory calibration consists of a polynomial function with 12 parameter values, which is applied to the raw values outside the sensor by the micro-controller. For the purpose of measuring the pressure distribution around an airfoil, however the factory calibration is not accurate enough. In order to increase the measurement accuracy, a calibration for each MEMS sensor was performed by determining an individual set of parameters for the factory polynomial function. The determination of the individual calibration parameters was performed with test runs in a pressure-temperature chamber with the temperature and pressure range required for the flight test. With this calibration for each sensor, the absolute accuracy could be increased from $1 \mathrm{hPa}$ to under $20 \mathrm{~Pa}$ over a temperature range from $-20{ }^{\circ} \mathrm{C}$ to $+30{ }^{\circ} \mathrm{C}$ and an absolute pressure range from $600-1100 \mathrm{hPa}$. The maximum ratings of this sensors make them very robust for flight testing. The maximum pressure from the data-sheet goes from $0 \mathrm{~Pa}$ to $20000 \mathrm{hPa}$, whereas the temperature range goes from $-45^{\circ} \mathrm{C}$ to $85^{\circ} \mathrm{C}$ [19]. During the calibration procedure and the flight test, pressures from $300 \mathrm{hPa}$ to $1200 \mathrm{hPa}$ and temperatures from $-40^{\circ} \mathrm{C}$ to $+60{ }^{\circ} \mathrm{C}$ were applied to each sensor without any abnormalities. The noise behavior of each sensor cannot be improved without intervention of the on-board filtering, which would in turn decrease the sample rate. However the remaining measurement noise of around $+/-6 \mathrm{~Pa}$ at 100 $\mathrm{Hz}$ is acceptable for the determination of flight loads.

For the flight test, four flexible Printed-Circuit-Boards (PCBs) were designed with 16 BMP280 sensors each, which are shown in green in Fig. 5. The flexible PCBs were manufactured, tested and calibrated. Due to test and time issues, the micro-controller for the PCBs was

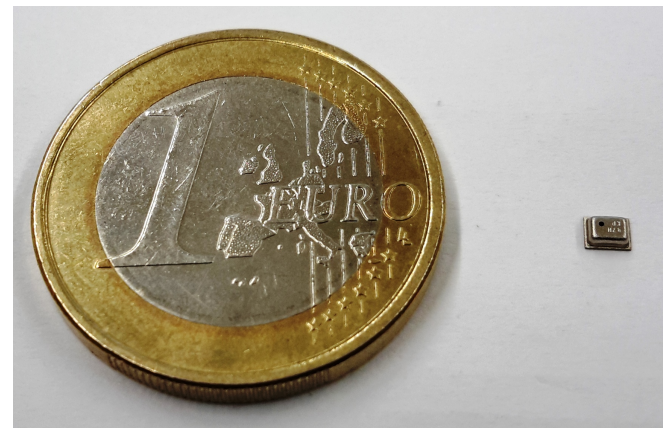

Fig. 3 Comparison in size of the BMP280 sensor and 1 Euro coin.

mounted outside of the wing glove. In Fig. 6 the micro-controller-box can be seen next to the wing glove. This micro-controller-box had the task, to aquire data from the two PCBs with 32 sensors on the top and to transfer the measured pressures to the data acquisition system at a sample frequency of $100 \mathrm{~Hz}$. A second box was mounted on the bottom side of the wing for acquiring data from the other 32 sensors on the airfoil bottom side.

\section{Wing Glove Construction}

In contrast to bigger aircraft where pressure belts can be used, on a glider with a chord length of less than $0.8 \mathrm{~m}$ and a mainly laminar boundary layer on the airfoil, it requires additional efforts to stay laminar while measuring. For this reason, a wing glove was built in the original shape of the main wing airfoil, but $12 \mathrm{~mm}$ thicker and $85 \mathrm{~mm}$ longer (scale factor 1.105), to get enough space for the circuit boards and cavities for the sensors shown in Fig. 4

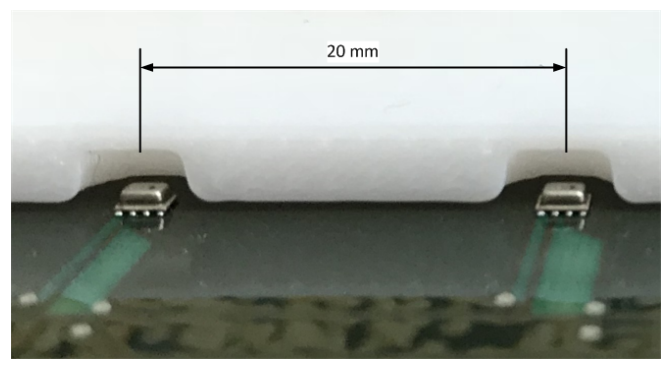

Fig. 4 Two BMP280 in a 3D-printed test part with cavities.
The glove consists of seven main mechanical parts, three aluminum ribs to ensure the shape of the surface, and four 3D-printed parts for the housing of the sensors. The 3D-printed parts were chosen, to integrate the PCBs with the sensors in a curved surface with small cavities for each sensor. The cavities are very small to reduce the viscous damping while measuring. Each cavity was carefully designed, because while gluing the PCBs, the glue was also used for sealing the cavities. The outer two 3D-printed parts were used for rounding the corners. To strengthen the glove and to smooth the 3D-printed surfaces, the wing glove was covered with glass fiber reinforced epoxy resin. Then the surface was sanded, painted and polished.

Finally, the holes connecting the sensor cavities with the surface were drilled with a diameter of $0.3 \mathrm{~mm}$, perpendicularly to the surface. The holes on the surface are oriented in a $15^{\circ}$ angle to the downstream direction to prevent influencing each other. In the end, the PCBs with the MEMS-sensors were glued in, with the sensors placed in the small cavities inside the glove. An example without glue is shown in Fig. 4. For this first flight test, the controllers for the circuit boards were placed in two extra housings, mounted beneath the wing glove, one for each side. In future 
applications the controller will be placed on each circuit board as well. The glove was mounted and fixed with aluminum tape on the right main wing. First test flights from stall speed to maneuvering speed showed no influence at all to the handling qualities of the aircraft. Therefore the speed range up to maneuvering speed was used in the test flights. In order to identify differences in the design in the actual flown wing glove geometry, the right wing with the mounted glove was scanned with an optical 3D metering system. The updated wing geometry led to a better agreement of the measured and the calculated pressure distribution with XFOIL explained later in Chapter IV]

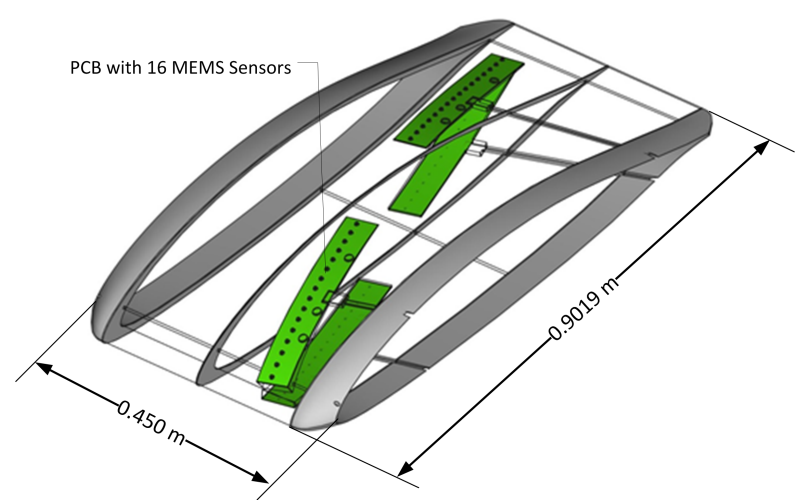

Fig. 5 Drawing of the wing glove with PCBs mounted.

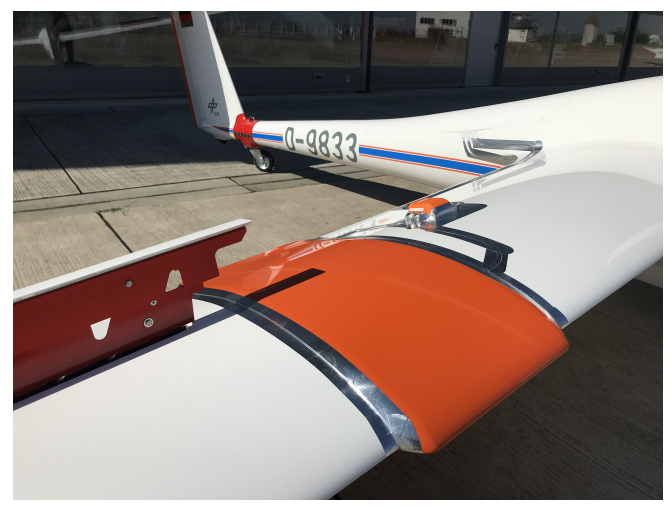

Fig. 6 Wing glove mounted on the wing for flight test.

The location of the MEMS pressure sensors and their status is depicted in Fig. 7 . As the number of sensors was limited to 32 on the profile upper and the lower surface, they were placed in regions of the profile were most of the changes in the pressure distribution would occur. All MEMS sensors were checked for their appropriate function after the integration into the wing glove. During this integration check it turned out that some sensor cavities were not sealed properly because the 3D-printed parts of the wing glove were not pressure tight. As a result of this check some corrupted sensors as shown in Fig. 7 were identified. Also, during flight No. 11 which was performed at the beginning of December, the pressure data of two sensors marked with red squares on the upper profile surface showed measurements that had stopped at a value above a certain height during climb at air temperatures well below $-10{ }^{\circ} \mathrm{C}$. In this case the sensor taps could have been sealed with frozen ice, because back on ground the sensors showed again a normal behavior.

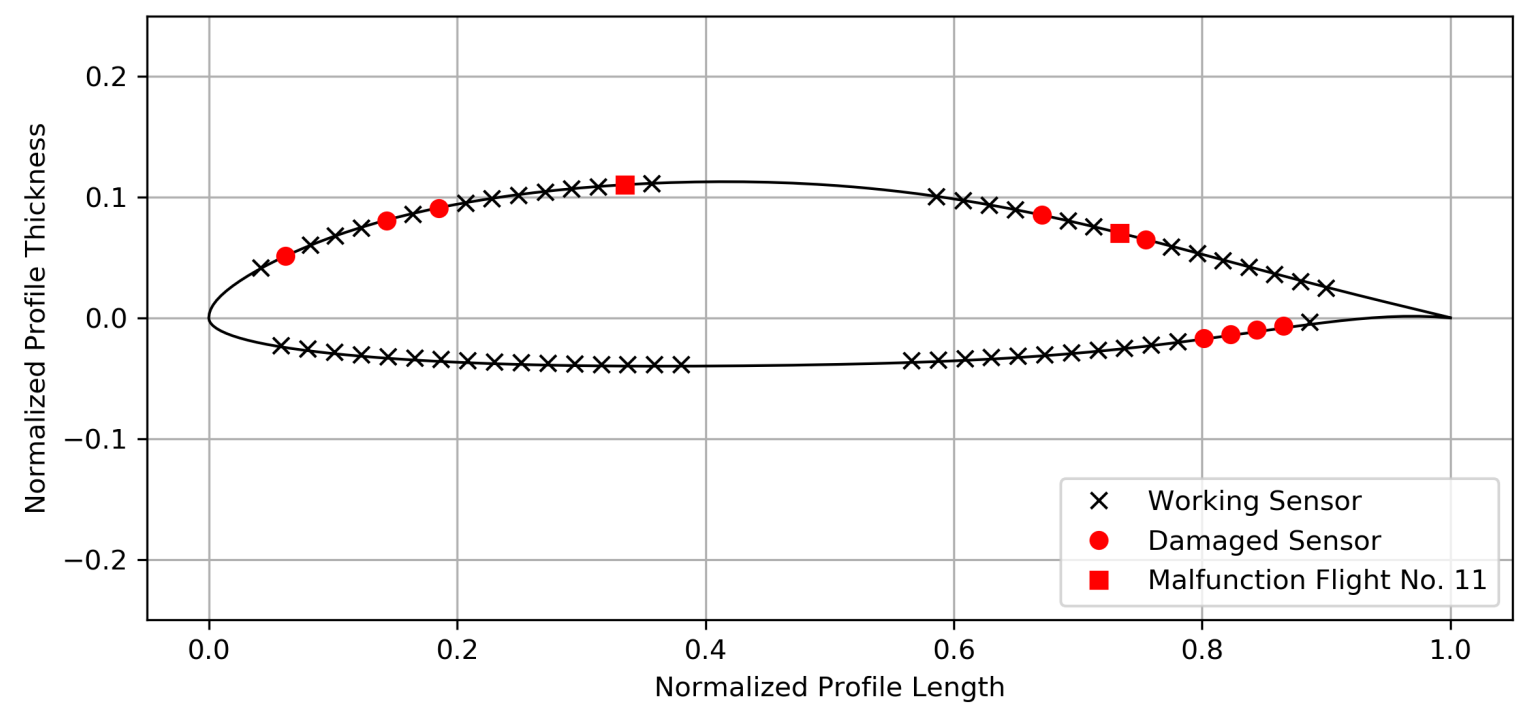

Fig. 7 Location and status of the MEMS pressure sensors. 
The measurement data of the faulty sensors were not taken into account in the subsequent investigations. In order to determine the appearance of the pressure distribution from the remaining sensor data with adequate accuracy, two fitting methods were developed, which are explained in chapter IV

\section{Flight Test Program}

The main scope of the flight test program was to investigate and compare the shear forces at the RH wing section measured with the SGs, with the ones determined from the measured pressure distribution around the wing glove. For this purpose flight maneuvers were defined with the intention to apply different varying aerodynamic loads on the aircraft structure.

The first group of test maneuvers contained steady flight conditions with static load factors in the z-direction of the aircraft body axes. The first steady maneuver was a wings level flight condition with a vertical load factor of $N_{z}=1$ where the aircraft was trimmed to a constant $V_{I A S}$ with a minimum change in velocity $\pm 2 \mathrm{~km} / \mathrm{h}$ and altitude $\pm 1.5 \mathrm{~m}$. This flight condition was maintained for at least 20 seconds. Steady level turn maneuvers at different bank angles were the second type of steady flight maneuvers. Starting from a stabilized wings level flight condition, the pilot initiated a roll maneuver to a specified bank angle. The pilot maintained a constant bank angle for a full circle and returned again to a wings level condition. The maneuver was repeated again to the opposite bank angle. The level turn maneuvers were performed with bank angles of $30^{\circ}, 45^{\circ}$, and $60^{\circ}$ imposing a theoretical vertical load factor of $N_{z}=1.2,1.4$, and $2.0 \mathrm{~g}$ on the aircraft.

A second group of flight maneuvers was defined with the intention to investigate dynamic load changes. Load changes with an $N_{z}<1 \mathrm{~g}$ could be established with a pull up - push over maneuver. Starting from a wings level condition the pilot applied a step wise input to the elevator in nose up direction. After 2 seconds he applied a step-wise input on the elevator in the nose down direction to achieve a constant vertical load factor. At a pitch angle of $-15^{\circ}$ he applied again a nose up elevator input with a constant load factor to return the aircraft to a stabilized horizontal flight condition. Other dynamic maneuvers included level turn maneuvers in combination with an elevator step input, stall as well as spoiler deployment and retraction during horizontal flight. Additional dynamic maneuvers were executed to ensure meaningful flight test data for data compatibility check (DCC), a methodology for checking and correction of the air data sensor and IMU signals. Besides the use for a DCC, measurements from these maneuvers were also of interest for the investigation of loads as they include dynamic load changes and extend the range of the measured AoA and AoS in the flight data set.

All maneuvers were performed at different initial speeds of 100,130, and $160 \mathrm{~km} / \mathrm{h}$. A total number of 11 flights were performed during the time of July and December 2018. All flights were carried out near the Braunschweig airport area in Germany. A test card was prepared for each flight, containing a set of maneuvers to be performed by the pilot. In order to minimize measurement disturbances, all flights were conducted in air with minimal turbulence and thermal updraft.

Before take-off the measurement equipment was turned on and the reception of sensor signals was checked. In a stand-still position on the ground, the aircraft was brought to a wings level condition and flight data was recorded for at least $10 \mathrm{~s}$. The measurements from this reference condition on ground was used later for the correction of pressure offsets. For each test flight the aircraft was towed to an altitude of about $3000 \mathrm{~m}$ above sea level, which took about half an hour. After releasing the glider aircraft at this altitude, the available flight time for test maneuvers was nearly another half an hour. The recorded flight was downloaded and saved to hard drive after landing. A total of 122 maneuvers were used for load investigation process.

The covered range of the flight envelope is shown in the diagrams of Fig. 8 The first diagram in the figure shows the measured vertical load factor $N_{z}$, plotted against $V_{I A S}$. A maximum load factor of $+4 \mathrm{~g}$ and minimum load factor of nearly $-0.4 \mathrm{~g}$ was achieved during the flight test maneuvers, indicating that the designed maneuvers led to a good coverage of different aircraft load conditions. During the flight test a maximum AoA of nearly $20^{\circ}$ was measured as can be seen in the second diagram of Fig. 8, where the AoA is plotted against the $V_{I A S}$. In the same way the AoS range which was covered with the flight test data, is presented in the third diagram, indicating that AoS values between nearly $-15^{\circ}$ and $+45^{\circ}$ have been achieved. 

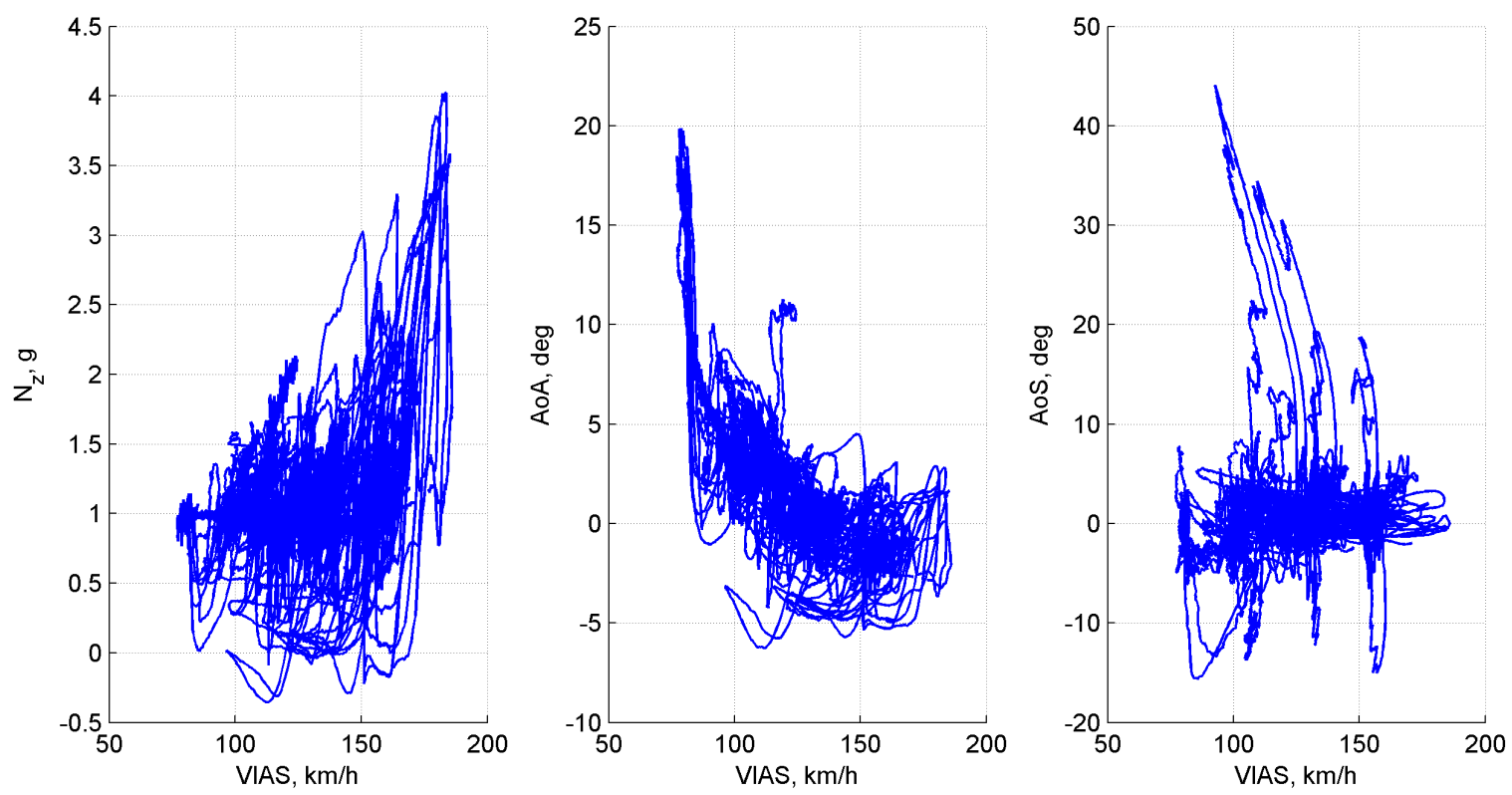

Fig. 8 Range of the measured flight test data.

\section{Data Analysis Process}

\section{A. Pre-Processing of Flight Test Data}

After the flight test the data from all flights were segmented into maneuver time slices. A data compatibility check (DCC) was performed on the air data and IMU measurements. The DCC is based on the integration of the measured accelerations and rotational rates of the IMU. Details about this methodology can be found in [20]. The results are reconstructed signals for $V_{T A S}$, AoA and AoS which can be compared to the measured air data values. That way correction parameters in terms of a factor and and an offset were identified for the AoA and AoS measured with the 5-hole-probe. During this process the correct function of the sensors for static and total pressure in the nose boom and the MEMS pressure sensors in the wing glove was checked.

As already mentioned in Chapter III.D before each flight a standstill condition was established on ground were the aircraft was leveled. During this reference condition, the dynamic pressure should be zero and the static pressure measured by the transducer in the 5-hole-probe should be equal to the values of the MEMS sensors in the wing glove. Measurement data from the standstill condition were used to check the pressure sensors and to calculate offset corrections, because the pressures measured at the nose-boom location were essential for the calculation of pressure distributions around the wing glove, later explained in Chapter IV.C

\section{B. Determination of Aerodynamic Wing Shear Force from SG Measurements}

The base for the comparison of the two load measurement methods is the shear force $F_{z, W l}$ of the RH wing part determined for load station No. 1, whose definition and position is shown in Fig. 9 As shown in the figure, the shear force $F_{z, W l}$ can be regarded as a point load which acts on the center of gravity $C G_{W l}$ of the wing section. Similar to Fig. 2 the location of the SG load station No. 1 and the wing glove is shown, as well as the origin of the reference coordinate system used for the definition of geometric positions and loads. The shear force at the RH wing load station No. 1 depicted in Fig. 2] was calculated from the SG gauge measurements using the load equations in report [17]. During the SG calibration process which led to the load equations, also the location of $C G_{W l}$ was determined by weighing of the different wing parts. The shear force measured by the SG at load station No. 1 is the result the of wing part weight, the inertial forces and the aerodynamic lift surface load as depicted in Fig 9 For the comparison of the shear force determined with the SGs and the force determined from the pressure distributions, it is necessary to extract only the part of the shear force from the SG measurements which is caused by aerodynamic loads. For this reason it was necessary to establish a $1 \mathrm{~g}$ reference condition, where the aircraft is unloaded and leveled on ground. Forces measured 


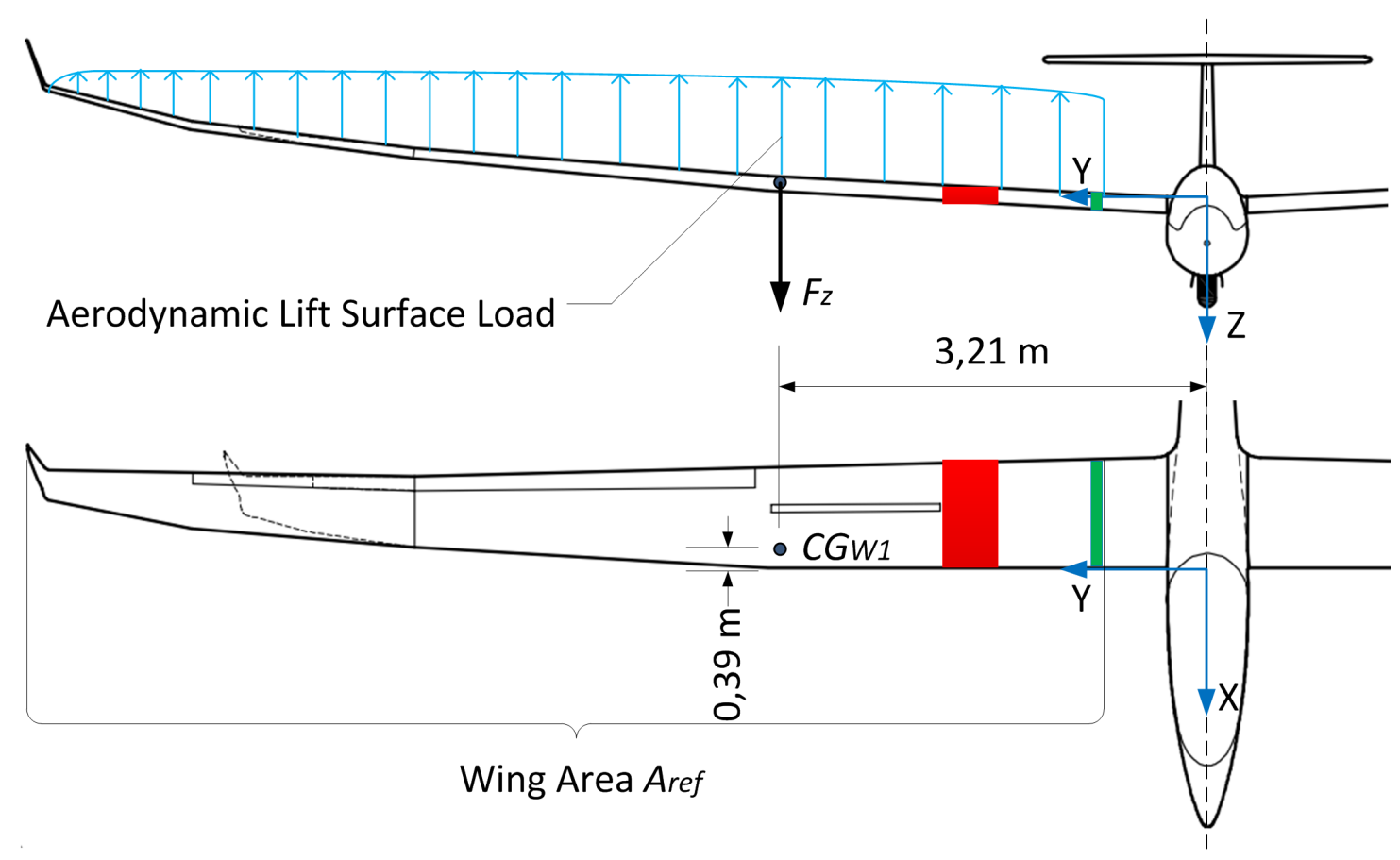

Fig. 9 Definition and location of RH wing aerodynamic shear force.

by the aircraft at the $1 \mathrm{~g}$ condition were caused by the weight forces of the structural components as well as aging effects of the SGs. For the determination of this offset, the Discus-2c was jacked in the hangar and the pitch and the bank angle were aligned to the zero level. At the leveled reference condition the SG measurements from $250 \mathrm{~s}$ were recorded and averaged. This identified offset $F_{z, S G, W l \text {, ref }}$ was subtracted from all SG measurements in order to get only the force caused by aerodynamic loads.

During flight however, the inertial forces change with the aircraft acceleration. For example during a constant turn maneuver with a $60^{\circ}$ bank angle, the inertial acceleration is $2 \mathrm{~g}$. For this reason, the inertial acceleration at the location of the RH wing part component CG must be taken into account in the calculation of the aerodynamic loads determined from the SG measurements. Using the distance between the RH wing component CG and the IMU $x_{C G_{W I}, I M U}, y_{C G_{W I}, I M U}, z_{C G_{W I}, I M U}$, the inertial acceleration in z-direction at the location of the wing component CG $a_{z, C G_{W I}}$ is determined with Eq. 1 from the acceleration and angular rate measurements of the IMU:

$$
\begin{aligned}
a_{z, C G_{W I}}= & a_{z, I M U}-\left(p_{I M U} \cdot r_{I M U}-\dot{q}_{I M U}\right) \cdot x_{C G_{W I}, I M U} \\
& -\left(q_{I M U} \cdot r_{I M U}+\dot{p}_{I M U}\right) \cdot y_{C G_{W I}, I M U}+\left(p_{I M U}^{2}+q_{I M U}^{2}\right) \cdot z_{C G_{W I}, I M U}
\end{aligned}
$$

The shear force $F_{z, S G, W l}$ at the wing load station caused by the aerodynamic loads is calculated from the SG measured shear force $F_{z, S G, W 1, m}$ and subtraction of the weight force at the $1 \mathrm{~g}$ condition $F_{z, S G, W l, r e f}$ and the force caused by the additional inertial acceleration component:

$$
F_{z, S G, W l}=\underbrace{F_{z, S G, W 1, m}}_{\text {SG Measurement }}-\underbrace{F_{z, S G, W 1, r e f}}_{\text {Weight Force at Ref. Cond. }}-\underbrace{m_{W 1} \cdot\left(a_{z, C G_{W l}}+g\right)}_{\text {Inertial Force }}
$$

In the last term of the above equation the normal earth acceleration is added to the acceleration determined with Eq. 1. because the IMU measurement contains already the $1 \mathrm{~g}$ earth acceleration component but with a negative sign. Therefore during a standstill leveled condition on ground with no aerodynamic forces acting on the structure, Eq. 2 is equal to zero. The mass of the RH wing part $m_{W l}$ was determined during the SG calibration campaign and amounts to $79.67 \mathrm{~kg}$. Equations 1 and 2 were applied to the measurements from the SGs at the RH wing load station No. 1. It should be noted that Eq. 1 is only valid for the assumption that the structure between the IMU and the wing part CG is rigid. In the 
underlying investigations the effects of flexibility were neglected, because only steady flight maneuvers were considered in the first evaluation of the load measurements.

\section{Calculation of Shear Force from Wing Glove Pressure Distributions}

In order to calculate the shear force from the local pressures measured by the MEMS sensors on the wing glove, the pressure distribution on the airfoil upper and lower surface had to be determined. In a first step the dimensionless pressure coefficient $c_{p}$ was calculated for each MEMS sensor with the following equation:

$$
c_{p}=\frac{p_{\text {local }}-p_{0}}{\frac{1}{2} \rho V_{0}^{2}}=\frac{p_{M E M S, m}-p_{s, N B, c o r}}{p_{d, N B, c o r}},
$$

where $p_{M E M S, m}$ is the pressure measurement of the individual MEMS sensor at the corresponding location on the wing glove, $p_{s, N B, c o r}$ is the static and $p_{d, N B, c o r}$ is the dynamic air pressure measured at the nose boom location. The static and the dynamic pressure measurements from the 5-hole-probe at the nose boom were corrected by the appropriate offsets, determined during the standstill condition on ground as explained in chapter IV.A The pressure coefficient $c_{p}$ was determined with Eq. 3 for each MEMS sensor using the averaged pressure measurements during the steady condition of the flight test maneuver. The dimensionless shear force coefficient $C_{z}$ was found by integrating the difference between the upper and lower pressure distribution over the normalized airfoil length $x_{c}$ according to Eq. 4 .

$$
C_{z}=\int_{0}^{1} c_{p, u p}\left(\mathrm{~d} x_{c}\right)-c_{p, l o}\left(\mathrm{~d} x_{c}\right) \mathrm{d} x_{c}
$$

The shear force $F_{z, M E M S, W l}$ acting on the RH wing part for the corresponding load section No. 1 was determined with Eq. 5 from the dynamic pressure $p_{d, N B, c o r}$, the dimensionless shear force coefficient $C_{z}$ and the reference area for the corresponding wing part $A_{\text {ref }, W l}$ :

$$
F_{z, M E M S, W I}=p_{d, N B, \mathrm{cor}} \cdot C_{z} \cdot A_{r e f, W I}
$$

The reference area used in Eq. 5 was estimated from the wing geometry data and considers only the RH wing part for load station No. 1 as shown in Fig. 9 Table 1 lists the geometric quantities being used for the calculation of the aerodynamic forces. It should be noted that this procedure for the shear force calculation assumes that the shear force i.e. the lift is equally distributed along the wing part. In reality this is not the case, because the outer thinner parts of the wing will produce less lift than the thicker inner parts. As the Discus-2c has a high aspect ratio, where the wing chord length stays constant over a great part of the wing span, this error in the calculation was neglected in the following considerations.

One essential point for the accuracy of the shear force calculated from the local pressures measured by the MEMS sensors is the correct determination of the pressure curves on the upper and lower side of the airfoil. As already explained in chapter III.C, the MEMS pressure sensors were not distributed equally along the chord length, because of the limited number of sensors and limited installation space in the rear sections of the wing glove airfoil. In addition, several sensors in the rear part of the airfoil showed a failure after installation. The lack of sensor measurements in the middle and rear section of the airfoil complicated the determination of the pressure curves from the measurements of the MEMS sensors.

Figure 10 shows the pressure curves for the upper and lower surface from the MEMS measurements: Here the results from a trimmed wings-level flight maneuver are shown. The black and white triangles show the averaged pressure coefficients calculated for each MEMS sensor with Eq. 3 for the respective upper and lower wing glove surface. The error bars on the triangles indicate the standard deviation for the pressure coefficients derived from the MEMS pressure measurements. In order to derive pressure distributions from these individual MEMS pressure measurements, a step-by-step procedure was developed and carried out:

1) The MEMS measurements were used to adjust an aerodynamic model for the pressure distribution around an airfoil. In this case the aerodynamic flow analysis program XFOIL was used. The pressure distribution determined with this optimization calculation is shown as a blue solid line.

2) Supporting points were generated with the pressure distributions determined with the XFOIL calculation, in the airfoil regions where no MEMS pressure measurements were available. These supporting points are indicated by red crosses in the mid and the rear parts of the airfoil. 
3) A polynomial fitting of the 12th order was applied to the MEMS pressure measurements, the calculated supporting points and a stagnation point at the beginning of the airfoil. The dashed black line represents the polynomial fit for the upper pressure distribution and the dashed dotted line represents the one for the lower surface pressure distribution.

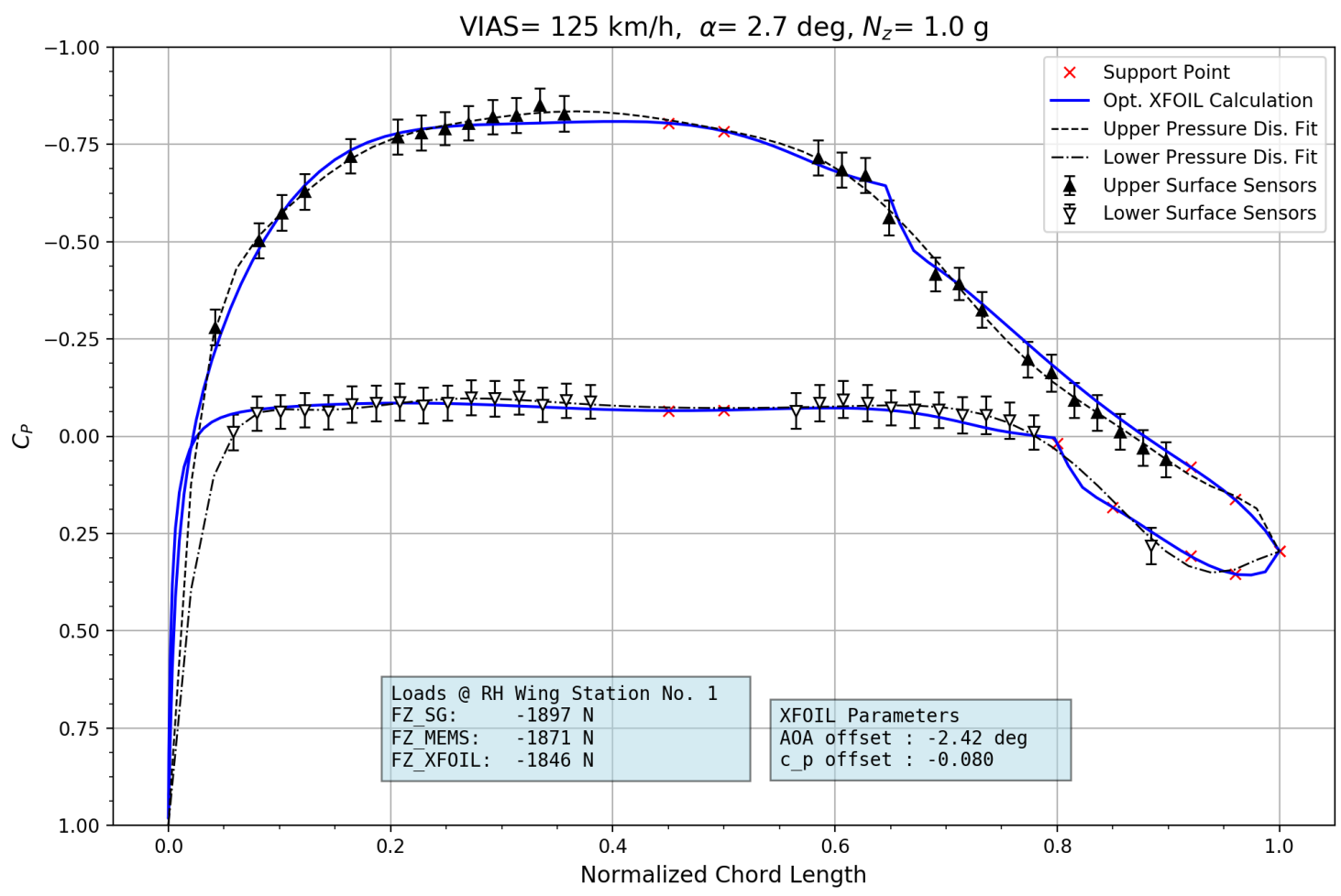

Fig. 10 Pressure distributions determined from MEMS measurements and an optimized XFOIL model calculation for trimmed level flight condition.

The XFOIL software uses a panel method with a correction for viscous flow to calculate the pressure distribution around a given airfoil geometry [21]. Besides the wing glove airfoil geometry, which was measured by an optical 3D scanning system, the other two inputs parameters for the XFOIL calculation were: AoA and Reynolds number. The Reynolds number was calculated from the air data measurements with the following equation:

$$
R e=\frac{V_{T A S} \cdot l_{c}}{v}
$$

With the listed input parameters, the XFOIL flow model calculated the pressure distribution on the upper and lower airfoil geometry. The calculated pressures at the location of each MEMS sensor were compared to the measured pressures. A least-squares optimization was performed in order to minimize the differences between both values. The result of this optimization is a fitted pressure distribution calculated by the XFOIL model, which is plotted in Fig. 10] as a blue line. Red crosses mark the supporting points determined with the XFOIL calculation for the polynomial fitting method.

An offset in the AoA measured by the 5-hole-probe was one of the parameters used in the optimization. This offset in the AoA may also be considered as an induced AoA caused by downwash effects of the 3-dimensional wing [22]. Therefore the effective AoA used as input for the XFOIL calculation was:

$$
\alpha_{e f f}=\alpha_{N B, m}-\alpha_{\text {ind }} \approx \alpha_{N B, m}+\alpha_{\text {offset,opt }}
$$

The second parameter for the optimization of the XFOIL model was a total offset in the pressure coefficients, calculated with the MEMS pressure sensor measurements. Having found an optimal fit for the XFOIL flow model, all MEMS 
based pressure coefficients were corrected with the optimized offset value according to the following equation:

$$
c_{p, M E M S}=c_{p, M E M S, m}-c_{p, o f f s e t, o p t}
$$

This correction caused a shift in the pressure coefficients derived from the MEMS pressure measurements in order to fit the XFOIL model calculation. As the shear force is calculated from the difference between the upper and lower pressure distribution, this shift has no impact on the shear force results.

The considerations for an adequate determination of the pressure curves on the upper and lower airfoil surface from the measurement data of the MEMS, finally led to two methods which were taken into account for the calculation of the shear forces in the following investigations: The first method uses a polynomial fitting function of 12th order, considering the MEMS pressure measurements, the supporting points determined with the optimized XFOIL calculation and the stagnation point at the airfoil front. As the optimized XFOIL calculation delivers as well pressure curves for the upper and lower surface, these resulting curves were used as a second method for the calculation of the shear force. It should be noted that although the XFOIL pressure curves are the results of a numerical method, they still depend on the MEMS pressure measurements, because the curves represent the best fit of these measurements. Equations 4 to 5 were applied to the respective pressure curves determined by both fitting methods. Integration of the pressure distribution curves lead to the shear force $F_{z, M E M S, W I}$ using the polynomial fitting method and the shear force $F_{z, X F O I L, W I}$ determined with the optimized XFOIL model calculation.

In Fig. 10 the difference between the shear forces calculated from the polynomial fitted and the XFOIL derived pressure curves is only $25 \mathrm{~N}$. In total this is a divergence of only $1.3 \%$ caused mainly by a different pressure distribution in the front region of the airfoil.

A difficulty occurred with the optimized XFOIL model calculations when applied to conditions with a high AoA and starting flow separation. This situation is shown in Fig. 11, where the measurements from a constant turn maneuver with a load factor $N_{z}$ of $2.0 \mathrm{~g}$ and a high AoA of $13^{\circ}$ are presented. The last 10 pressure measurements located at the rear end

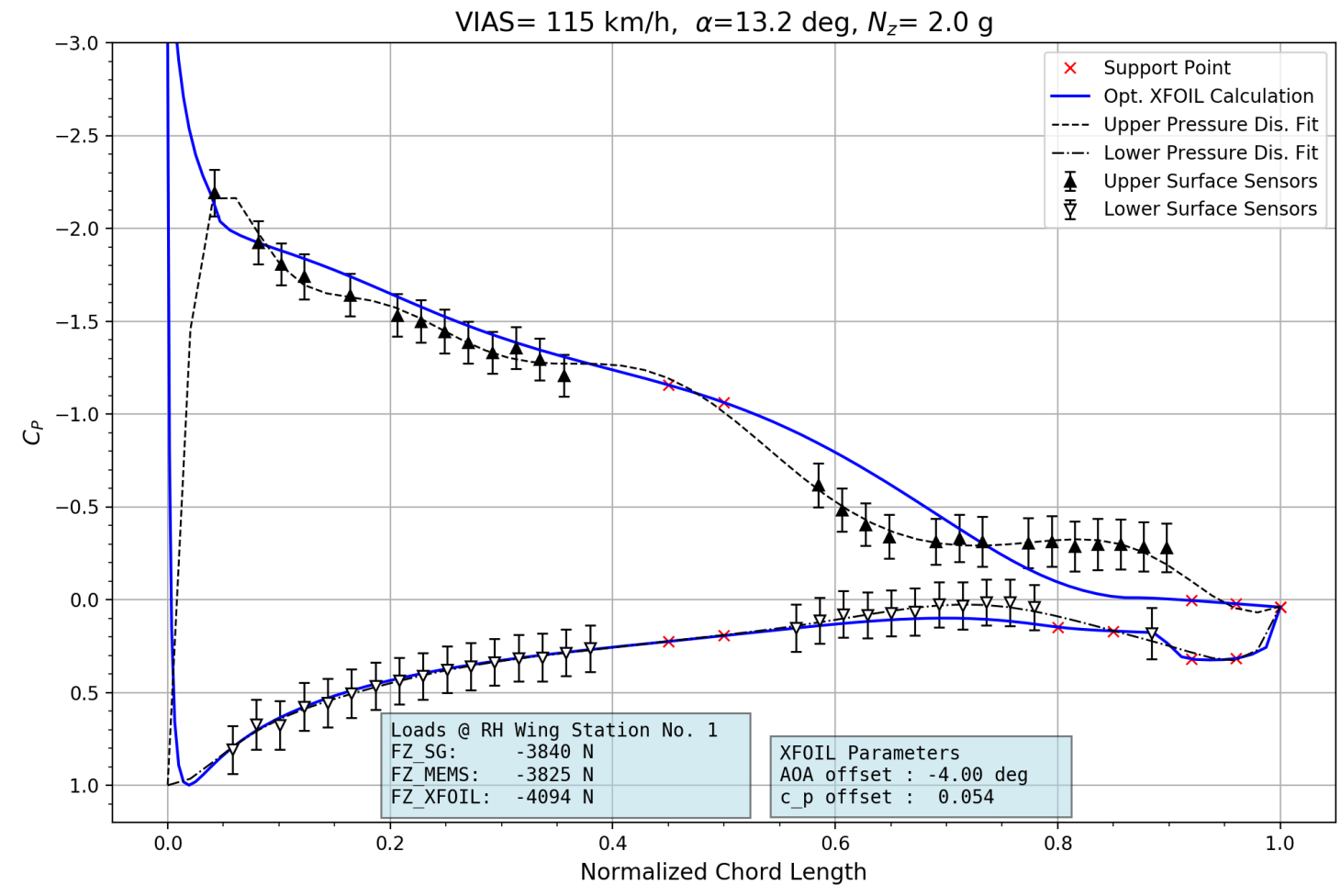

Fig. 11 Pressure distributions determined from MEMS measurements and an optimized XFOIL model calculation for a constant turn near stall. 
of the airfoil indicate a nearly constant pressure coefficient, a sign for the beginning of flow separation. They also deviate significantly from the pressure distribution calculated with the XFOIL optimization, represented by the blue solid line.

Whereas the pressure curve on the lower surface is in quite good accordance with the MEMS sensor pressure coefficients, the pressure curve on the upper surface shows large offsets to the MEMS pressure measurements. Especially in the front section of the airfoil the XFOIL calculation tends to overestimate the pressure coefficient under these flow conditions.

The different pressure distributions of the XFOIL model and the fitted MEMS measurements led to a divergence in the calculated shear forces of $267 \mathrm{~N}$. Considering the absolute values of the shear forces, the one determined with the XFOIL calculation is $7.5 \%$ higher than the one determined with the polynomial fitting method. The polynomial fitting method, taking into account the MEMS measurements and the supporting points from the XFOIL calculation, delivers resulting shear forces which are more consistent with the prevailing flow conditions. For this reason both methods for the calculation of pressure distribution curves from the MEMS measurements were investigated in the further analysis of flight loads.

\section{Results and Discussion}

The flight test program with the Discus-2c glider aircraft included maneuvers with static as well as dynamic load changes. For this paper, however in a first attempt only steady and quasi-steady maneuvers were evaluated and the results are presented in the following sections.

\section{A. Trimmed Wings-Level Flight Maneuvers}

The flight loads investigation started with a trimmed wings-level flight condition at $N_{z}=1 \mathrm{~g}$. In this case the aerodynamic lift is nearly equal to the aircraft weight force. The maneuver time slices with a trimmed wings-level flight condition had a duration of 8 to $25 \mathrm{~s}$ and had to fulfill the following criteria: The pitch and roll angle variation should be within a limit of $\pm 3^{\circ}$, the average vertical load factor $N_{z}$ during the maneuver should be $1 \mathrm{~g}$ with a maximum variation smaller than $\pm 0.1 \mathrm{~g}$. Having applied these criteria to the data set, a total of 14 trimmed wings-level flight maneuvers were selected for further investigations.

For all maneuvers, the resulting shear force at the RH wing as depicted in Fig. 9 was determined. For each maneuver time period the averaged measurements of the MEMS sensors and SGs were taken and processed according to the method described in Chapter IV] Figure 12 shows the shear forces determined with the different measuring methods for each wings-level flight maneuver. Black triangles indicate the SG measurements, the blue crosses indicate the results where the polynomial fitting method was used for the calculation of the pressure curves, the red dots indicate the results where the optimized XFOIL model method was applied. In order to examine the dependency of the results on the airspeed, a linear regression was applied to the measurements. The resulting functions are plotted in the diagram as a black solid line for the SGs, as a blue dashed line for the polynomial fitting method and as red dashed dotted line for the optimized XFOIL model method. In the diagram legend, the equations of the linear fitting functions are presented. It includes the standard error $S E$ and the squared correlation coefficient $R^{2}$ for each fitting function, allowing an evaluation of the quality of the applied linear regression.

For each individual maneuver the shear forces determined by the three methods are not too far apart. When compared to the SG measurements, the shear forces using the polynomial fitting show a maximum deviation of $150 \mathrm{~N}$. The maximum deviation is smaller for the shear forces determined with the optimized XFOIL calculation method, which is around $100 \mathrm{~N}$. The maneuvers flown around $130 \mathrm{~km} / \mathrm{h}$ show the smallest deviation between the forces. In general the shear forces measured with the SGs have the tendency to be smaller than the ones determined from the pressure measurements. Concerning the linear fitting functions, the shear forces determined with the pressure distribution methods show a significant negative slope, indicating a dependency on the airspeed $V_{I A S}$.

One reason for this behavior could be an approximation error, which is caused by extrapolating the pressure distribution measured around the wing glove over the entire wing. Another source for errors is the wing part area $A_{\text {ref }, W l}$ used in Eq. 5. This area was determined roughly from a technical drawing of the wing. In reality the reference area used for the calculation of the shear force might be a little smaller. 


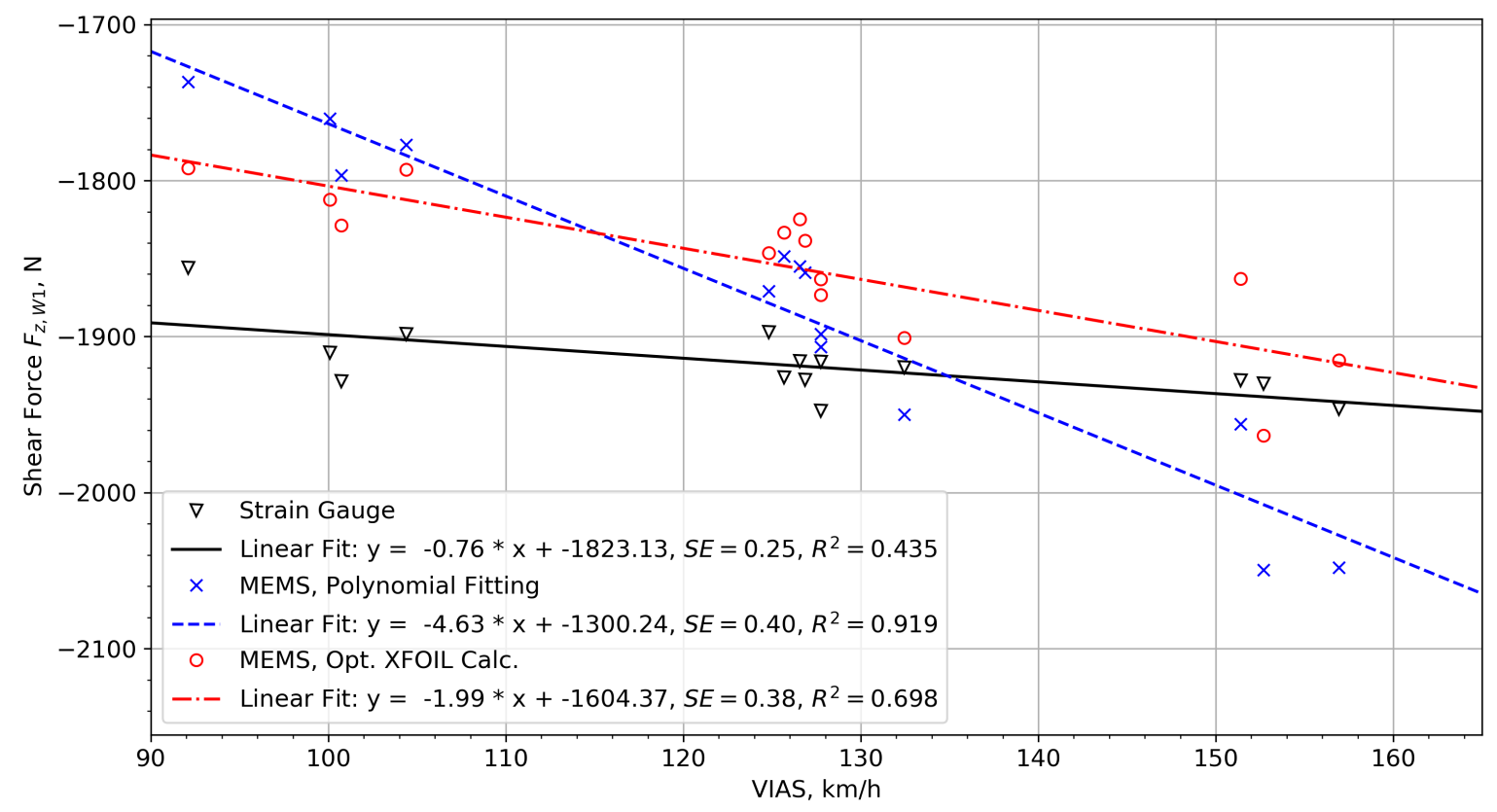

Fig. 12 Shear forces determined with the SGs measurements and from the MEMS pressure distributions for each trimmed wings-level flight maneuver plotted vs. $V_{I A S}$.

The standard error of the linear regression function is a measure for the variance of the shear force values around the trend line. Here the standard error for the SG measurements is lower than the one determined for the MEMS pressure distribution methods, which both have similar values. The variation of the shear force measurements is not only influenced by sensor noise alone, but also depends on the individual flight test condition. For example during one maneuver, a constant wings-level horizontal flight condition might have been easier to maintain than during other maneuvers. In order to investigate the issue of measurement variations further, the $100 \mathrm{~Hz}$ flight data of three selected maneuvers was averaged to a sample rate $25 \mathrm{~Hz}$ and for each sample the shear forces were determined. The results of this process are presented in Table 2 . For each of the selected maneuvers the total recording time, the average values for $V_{I A S}$ and the shear forces $F_{z}$ are presented together with their standard deviation. The $V_{I A S}$ has a very low standard variation, indicating that the speed was well maintained during the maneuvers. The shear forces determined by three measurement methods all have a standard deviation in a similar range of 10 to $22 \mathrm{~N}$, indicating that all three methods have comparable measurement variation characteristics.

For a better assessment of the practical applicability of the MEMS based flight load determination method, the relative deviation of the shear force determined the with pressure distribution measurement from the ones measured with the SGs was calculated according to the following equation:

$$
f=\left(\frac{F_{z, M E M S} / \text { XFOIL }}{F_{z, S G}}-1\right) \cdot 100 \%
$$

The results for each wings-level maneuver are presented in Fig. 13 .

Table 2 Analysis of force measurement variation for selected trimmed wings-level maneuvers.

\begin{tabular}{cccccccccc}
\hline ID & Duration, $\mathrm{s}$ & $V_{I A S}, \mathrm{~km} / \mathrm{h}$ & $\sigma_{s}$ & $F_{z, S G}, \mathrm{~N}$ & $\sigma_{s}$ & $F_{z, M E M S}, \mathrm{~N}$ & $\sigma_{s}$ & $F_{z, X F O I L}, \mathrm{~N}$ & $\sigma_{s}$ \\
\hline M1-04 & 10 & 92 & 0.045 & -1856 & 10.1 & -1737 & 10.0 & -1792 & 10.1 \\
M1-12 & 20 & 127 & 0.038 & -1916 & 17.3 & -1855 & 21.1 & -1825 & 22.2 \\
M1-01 & 12 & 151 & 0.087 & -1925 & 12.8 & -1948 & 20.9 & -1856 & 18.0 \\
\hline
\end{tabular}




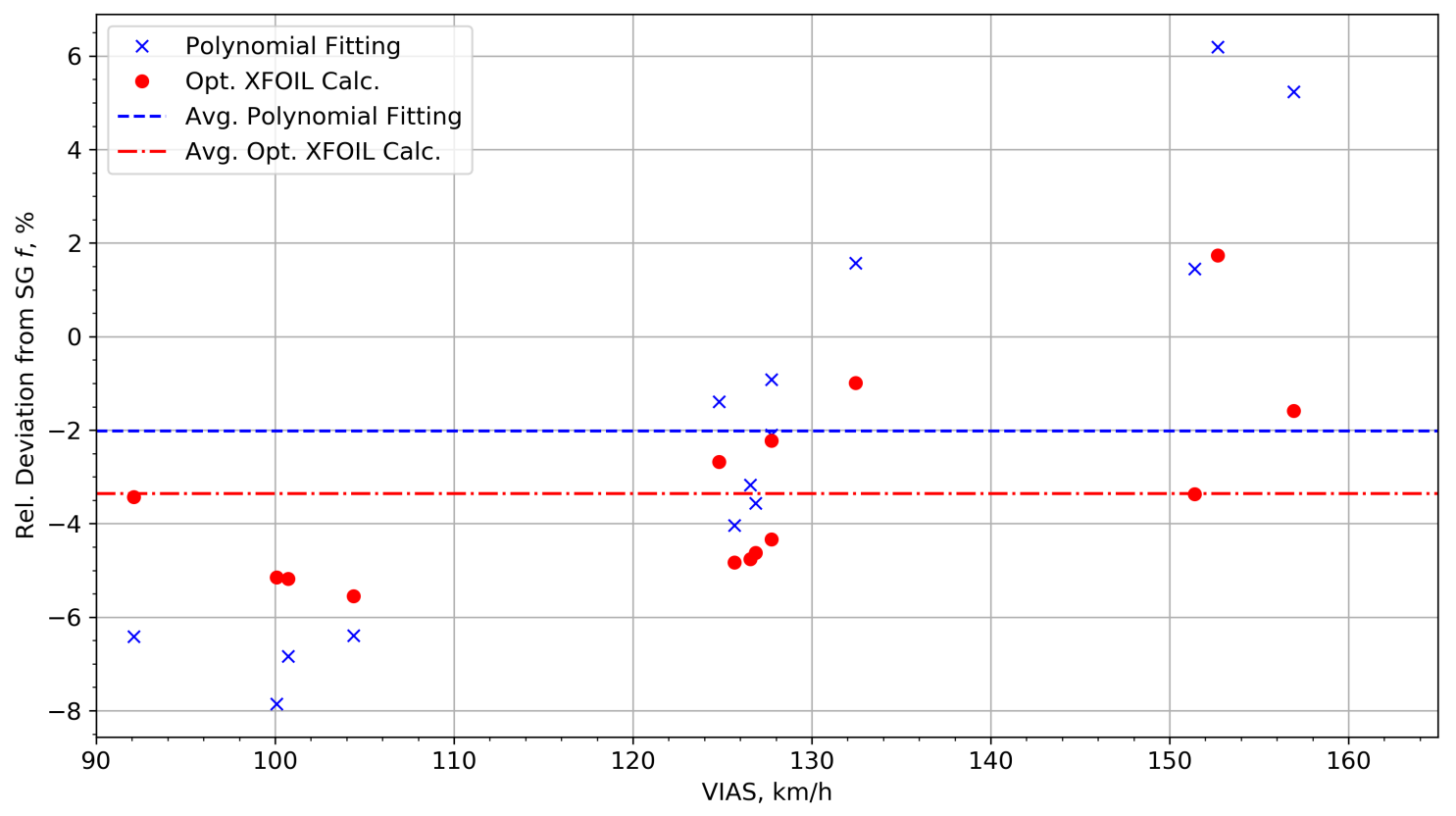

Fig. 13 Relative deviation of the shear forces based on MEMS pressure measurements from the ones determined with the SGs for each trimmed wings-level flight maneuver plotted vs. $V_{I A S}$.

The deviations for the shear force determined with the polynomial fitting method, marked by the blue crosses range from -7.9 to $+6.3 \%$. With values ranging from -5.6 to $+1.7 \%$ the deviations for the shear force using the optimized XFOIL model method are smaller. Taking into account all results from the trimmed wings-level flight maneuvers the blue dashed and the red dashed dotted line represent the average deviation for the respective shear force calculation method. Although the values derived with the polynomial fitting method method have a larger variation than the ones derived with the optimized XFOIL calculation, the total average is much smaller. In that case the total average is - $2.0 \%$ compared to the $-3.4 \%$ of the XFOIL method. As already mentioned before, a large part of the deviations result from the assumption that the aerodynamic lift force acts uniformly on the wing. Nevertheless, the shear forces determined from the MEMS pressure measurement are in a qualitative good accordance compared to the ones determined with the SGs.

\section{B. Constant Turn Maneuvers}

In order to impose loads higher than $1 \mathrm{~g}$ on the aircraft structure, constant turn maneuvers with different bank angles were performed during the flight test. As already mentioned in Chapter III.D all turn maneuvers were flown at similar speeds as the trimmed wings-level flight maneuvers. From the recorded time series data a total of 34 constant turn maneuver time slices were selected with a nearly steady flight condition and a duration between 5 and $27 \mathrm{~s}$. The time slices were cut and the measurements were averaged in order to minimize any divergence from the steady condition. An average vertical load factor $N_{z}$ between 1.1 and $2.0 \mathrm{~g}$ was achieved during the flight test maneuvers. For all individual maneuvers the bank and pitch angle have a maximum variation of not more than $\pm 3^{\circ}$. However, in some cases the vertical load factor had a maximum variation of $\pm 0.1 \mathrm{~g}$ especially at turns with bank angles larger than $50^{\circ}$. This was caused by the challenge of flying a constant turn in a glider aircraft: During the maneuver the pilot has to pull constantly at the elevator to minimize changes in the altitude. This is especially difficult at high load factors and high AoAs. The turn maneuver corresponding to Fig. 11] was especially challenging as the aircraft was operated near stall, indicated by a high AoA of nearly $13^{\circ}$.

The shear forces determined with the MEMS pressure sensors and the ones determined with the SGs for each constant turn maneuver are presented in Fig. 14 Here, the shear force for each maneuver is presented for the all three load determination methods and plotted versus the average vertical load factor $N_{z}$. For each method a linear regression was performed on the shear force values. The resulting linear fitting function and its characteristics are shown in the 
diagram legend.

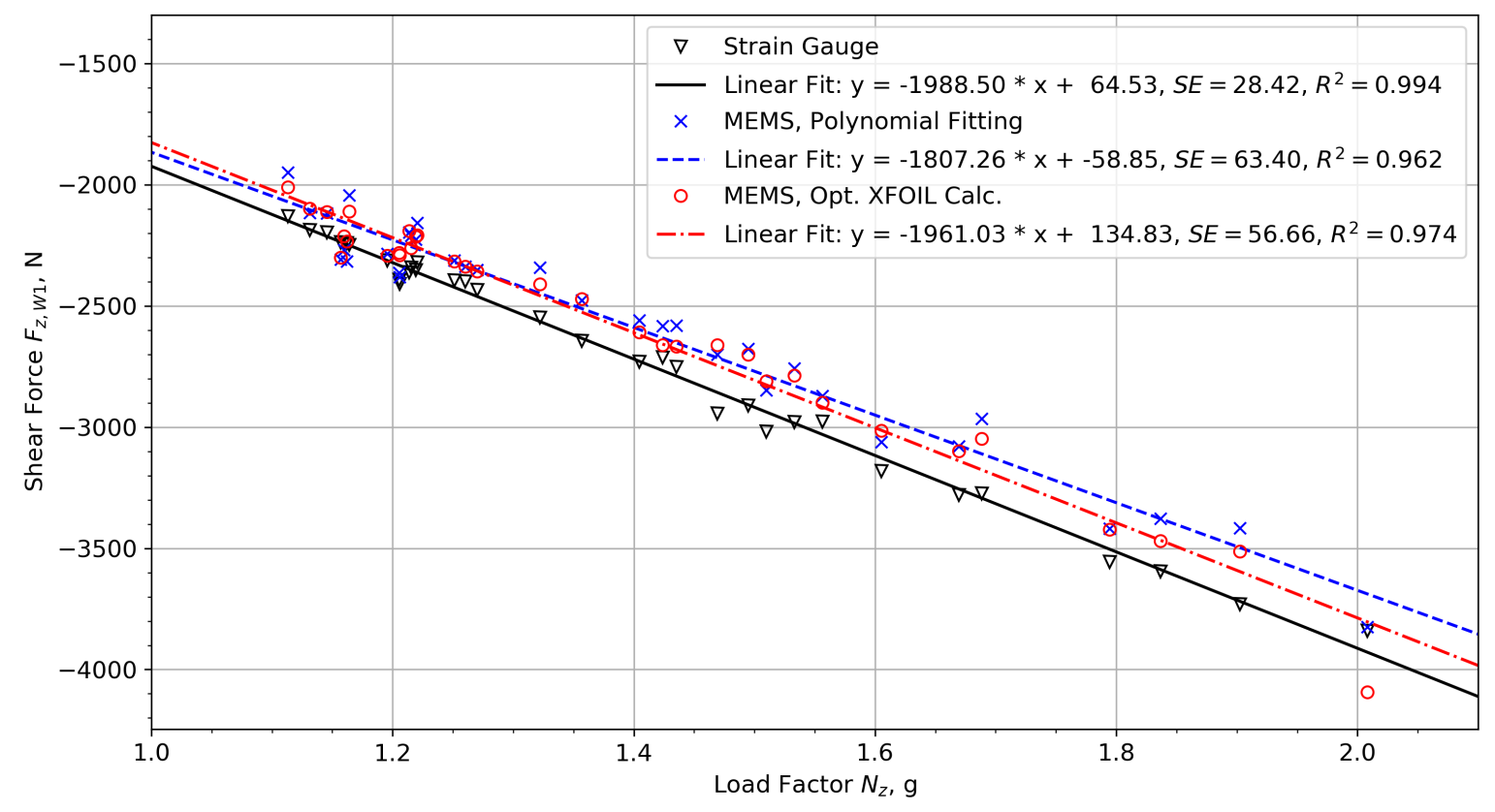

Fig. 14 Shear forces determined with the SGs measurements and from the MEMS pressure distributions for each constant turn flight maneuver plotted vs. $N_{z}$.

In general the shear forces from the SG measurements and the ones derived from the pressure distributions are close together and show no large deviations. In the case of the turn maneuver with $N_{z}=2.0 \mathrm{~g}$, however the shear force determined by the optimized XFOIL calculation method shows an offset of more than $250 \mathrm{~N}$ to the shear force value of the SGs and the one derived with the polynomial fitting method. Here the weakness of the XFOIL model becomes visible to reproduce the flow conditions correctly where the aerodynamic flow starts to separate. The fact that this method is not suitable for flow conditions with high AoA was already mentioned in in Chapter[IV] where the pressure distributions for the maneuver with $2.0 \mathrm{~g}$ was depicted in Fig. 11

When comparing the linear fitting functions for each shear force determination method, all have very similar slopes with a negative sign. The slope for the SG measurements is, however slightly steeper and the SG measurements have the tendency to measure higher absolute shear force values when compared to the ones determined from the pressure distributions. A cause for this discrepancy could be, as already mentioned earlier, the error made during the extrapolation of the measured pressure distribution around the wing glove over the entire RH wing part. Another issue is the structural flexibility: With increasing load factor, twisting and bending of the wing increases, leading to a more non-uniform lift distribution. Deviations can also be found in the offsets of fitting functions for the three load measuring methods. The reason for this characteristic can be manifold: In addition to the missing ability of the measurement setup to capture all aerodynamic effects, remaining calibration errors and temperature effects may have an influence on the measurements here.

A comparison of the standard error of the three linear regression functions shows, that the one for the SGs is almost $50 \%$ smaller than the ones for the pressure distribution methods. This higher variance of the shear force values, determined with the pressure distributions, was investigated further like in the previous section. The recorded measurements of three selected maneuvers were averaged to a sample rate of $25 \mathrm{~Hz}$ and the shear force values were determined for each sample. Table 3 presents the results of this evaluation: For each maneuver the duration of the evaluated time slice and the averaged values of the load factor $N_{z}$ and the shear forces $F_{Z}$ are listed. Concerning the standard deviations of all three measuring methods, the values tend to be higher than for the trimmed wings-level maneuvers. The standard deviation for the shear forces determined from the pressure distributions are however almost $50 \%$ higher than the ones determined with the SGs. For all three methods the standard deviations increase with 
Table 3 Analysis of force measurement variation for selected trimmed constant turn maneuvers.

\begin{tabular}{cccccccccc}
\hline ID & Duration, $\mathrm{s}$ & $N_{z}, \mathrm{~g}$ & $\sigma_{s}$ & $F_{z, S G}, \mathrm{~N}$ & $\sigma_{s}$ & $F_{z, M E M S}, \mathrm{~N}$ & $\sigma_{s}$ & $F_{z, X F O I L}, \mathrm{~N}$ & $\sigma_{s}$ \\
\hline M7-08 & 10 & 1.16 & 0.01 & -2248 & 16.2 & -2044 & 30.0 & -2109 & 34.2 \\
M8-08 & 20 & 1.32 & 0.01 & -2548 & 25.0 & -2342 & 50.5 & -2410 & 55.8 \\
M9-04 & 12 & 1.90 & 0.08 & -3730 & 143.9 & -3417 & 155.1 & -3509 & 169.0 \\
\hline
\end{tabular}

increasing vertical load factor of the maneuver. In case of the SG measurements for example the standard deviation is $16.2 \mathrm{~N}$ for the maneuver with the load factor of $1.16 \mathrm{~g}$. For a turn maneuver with a load factor of $1.90 \mathrm{~g}$, the standard deviation is much higher and amounts to $143.9 \mathrm{~N}$. These differences between the maneuvers are mainly caused by the challenge to maintain steady and calm flight conditions during the maneuver, especially with a bank angle higher than $50^{\circ}$. The variation of the measured values is therefore higher during maneuvers with higher load factor. This is also indicated by the standard deviation of the load factor listed in Table 3 which is much higher in the case for the maneuver with the $N_{z}=1.90 \mathrm{~g}$.

The relative deviation of the shear forces based on the pressure measurements from the ones measured by the SGs for the constant turn maneuvers was calculated according to Eq. 9 and is shown in Fig. 15. Here again the discrepancy in case of the turn maneuver with a vertical load factor of $N_{z}=2.0 \mathrm{~g}$ becomes visible. The shear force determined with the optimized XFOIL calculation method diverges $+6.6 \%$ from the one determined with the SGs, whereas the result determined with the polynomial fitting method has only a diversion of $-0.4 \%$.

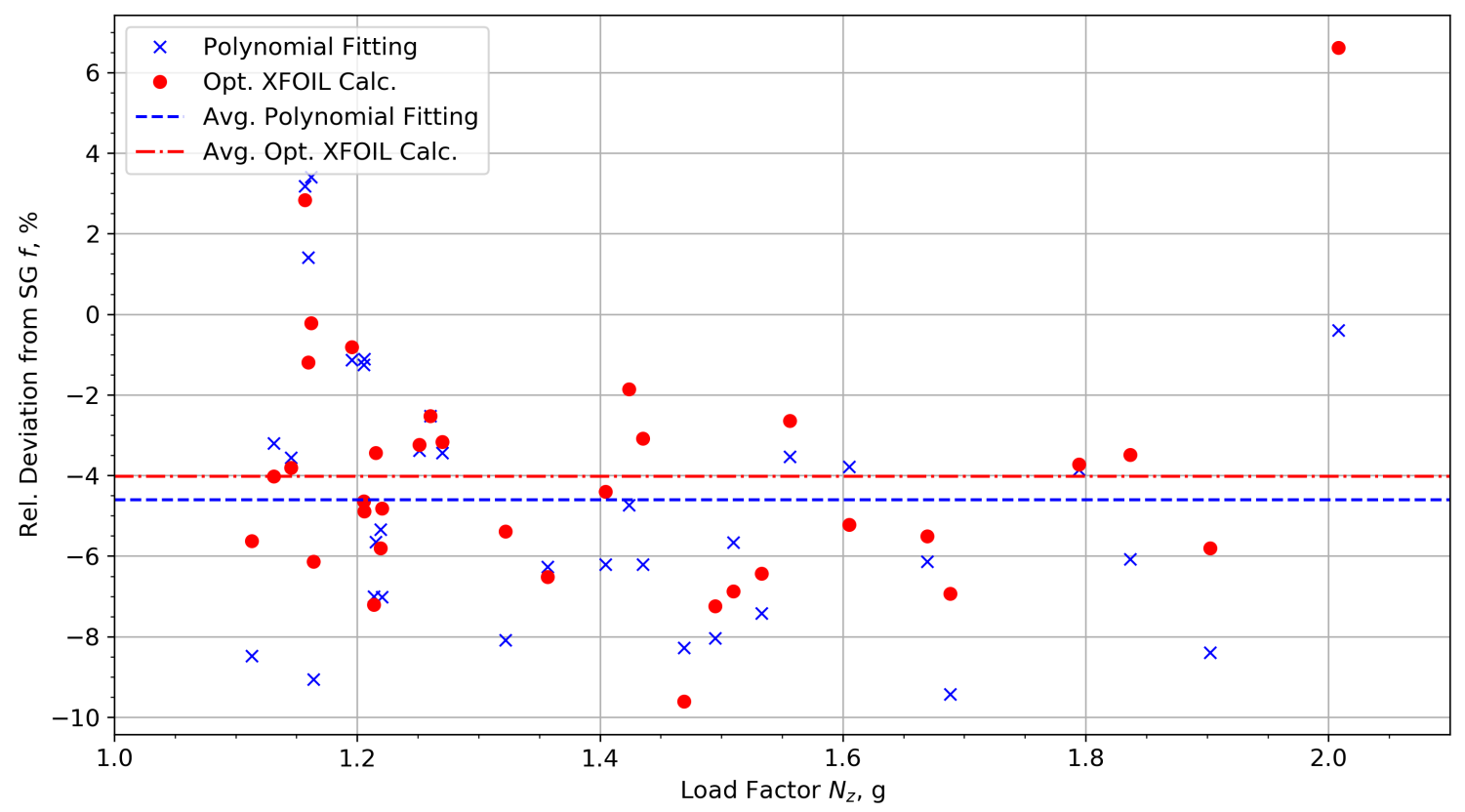

Fig. 15 Relative deviation of the shear forces based on MEMS pressure measurements from the ones determined with SGs for each constant turn flight maneuver plotted vs. $N_{z}$.

Considering all turn maneuvers the total average of the deviations are presented in the diagram of Fig. 15 as a blue dashed and a red dashed dotted line according to the respective pressure curve determination method. The results determined with the optimized XFOIL model calculation show an average deviation of $-4.0 \%$, the ones derived with the polynomial fitting method have an average deviation of nearly $-4.3 \%$. 


\section{Summary}

For a joint evaluation, the results from the trimmed wings-level and the constant turn flight maneuvers were merged together. In Fig. 16 the shear forces $F_{z, w 1}$ for the RH wing part determined by the SGs measurements, the MEMS pressure distribution using the polynomial fitting and ones determined with the optimized XFOIL calculation method are shown and plotted against the vertical load factor $N_{z}$. As already mentioned in the previous section the shear force determined by the XFOIL calculation method at $N_{z}=2.0 \mathrm{~g}$ shows a large discrepancy because of the beginning of flow separation. This value was discarded in the plot and was not taken into account for the linear regression calculation of the shear force values. The parameters for the linear fitting functions are again presented in the legend of the diagram.

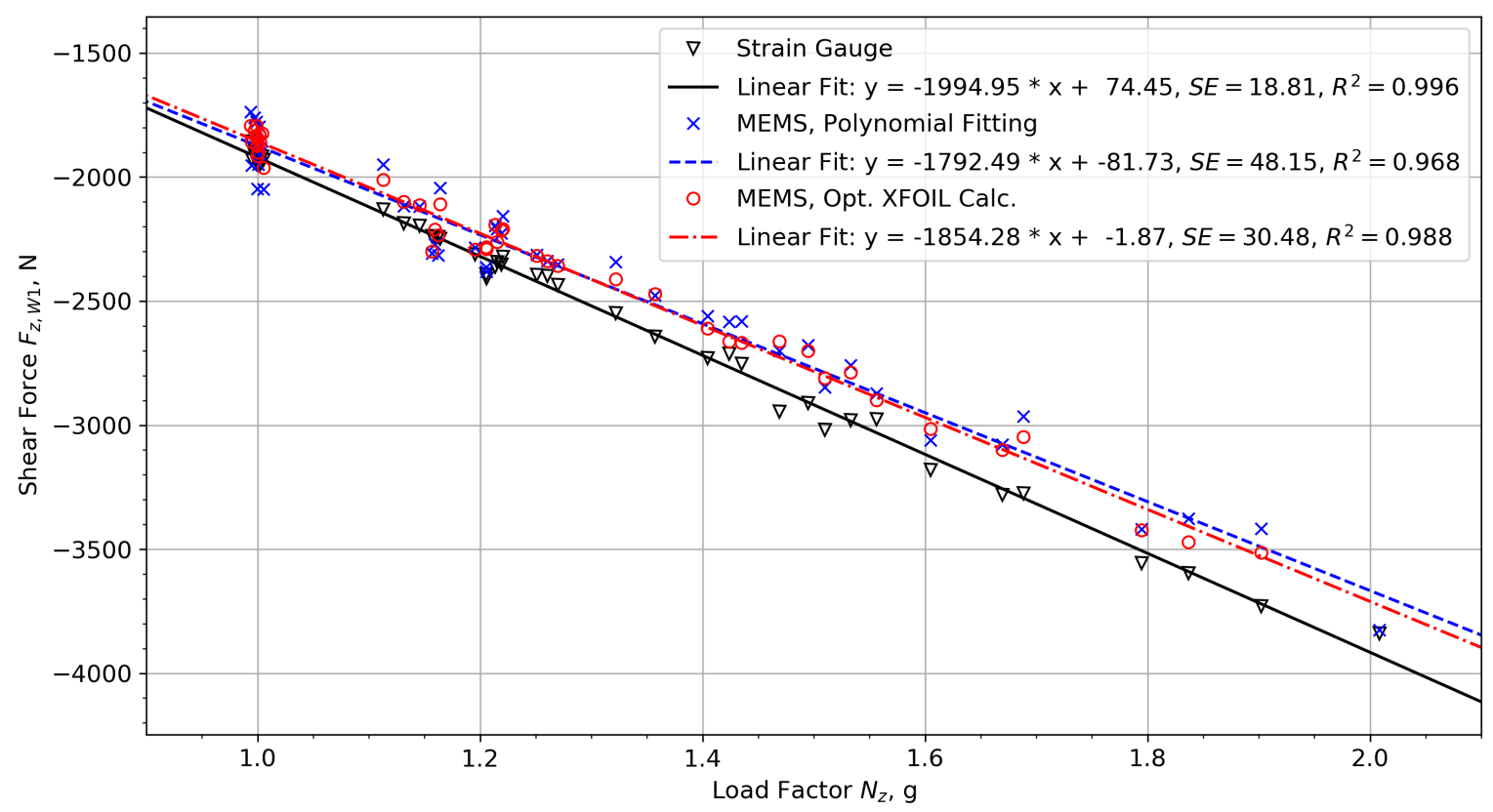

Fig. 16 Shear forces determined with the SGs measurements and from the MEMS pressure distributions for all wings-level and constant turn maneuvers plotted vs. $N_{z}$.

The overall evaluations show that the shear forces determined with the MEMS pressure measurements do not differ too much from the ones determined by the SG measurements. The SG method tends to determine much higher vertical shear forces in absolute values than the pressure based method. Considering the standard error of the linear regression functions determined for all three methods, the shear forces determined by the pressure based methods have a much higher variation. A large part of this variation is caused by the practical flight test conditions, which are almost never perfectly steady. Taking into account all results, the relative deviation from the SG values for both pressure based shear force determination methods is around $-4 \%$. As the SGs have a measurement accuracy of $\pm 3.6 \%$, for the validation loads used during calibration, the comparison alone does not reveal, which of the measurement approaches is better or worse.

The results show, however that the MEMS pressure sensor method for the determination of flight loads delivers at least results within the same accuracy range as the strain based flight loads determination method. Some proposals for the improvement of the measurement accuracy of the pressure sensor based load determination have already been mentioned:

- Increasing the local resolution of the pressure measurements by the instrumentation of several wing sections along the span with MEMS pressure sensors. This should enhance the determination of the exact shape of the lift distribution on the wing.

- A more accurate determination of the wing part reference area used for the determination of the shear force by considering e.g. the 3D-scanned wing geometry. 
The accurate determination of the pressure distribution around the wing airfoil is essential for the quality of the shear force calculated from the MEMS pressure sensor measurements. One way to improve the fitting quality of the pressure measurements would be to increase the number of sensors, especially in the front and the upper rear section of the wing glove airfoil. Due to the very short manufacturing process nine sensors on the wing glove showed corrupted measurements, because of a leaky bonding of the sensor cavities. This insufficient sensor sealing could be avoided in future setups with an improved manufacturing process.

\section{Conclusion and Outlook}

The potential use of MEMS pressure sensors for the determination of aerodynamic loads acting on the aircraft structures was investigated. Flight tests with the DLR research glider aircraft Discus-2c were performed. This aircraft was equipped with a calibrated strain gauge system for measuring shear force, torsion and bending moments at dedicated sections of the wing, fuselage and tail. It provided a suitable test platform for the comparison of two ways for the determination of flight loads: The conventional method based on strain gauge measurements and a method based on the pressure distributions determined with MEMS sensor measurements. A wing glove with a total of 64 pressure sensors installed along the upper and lower airfoil surface was constructed and placed on the RH wing. Additionally, the Discus-2c was equipped with a 5-hole-probe system, which provided air data measurements and an IMU platform for measuring inertial accelerations and rotational rates. During the flight test different dynamic and steady maneuvers with varying vertical load factors were performed. The MEMS sensors provided reliable local pressure measurements during the flight. These local pressure measurements were fitted to pressure distribution curves on the airfoil upper and lower side to determine an aerodynamic shear force for the wing part section. This force was compared to the corresponding shear force calculated from the strain gauge measurements at the RH wing load station.

For the comparison of both load determination methods, the flight data recordings of two steady flight maneuvers, trimmed wings-level flight and constant turn, were analyzed and evaluated. In general the shear forces determined with the pressure measurements were in good accordance with those determined from the strain gauge measurements. Taking into account all analyzed steady flight maneuvers, the shear forces determined with the MEMS pressure measurements deviate around $-4 \%$ from the shear forces calculated with the strain gauge measurements. The flight load determination method based on the MEMS pressure measurements is able to deliver results within the same quality and reliability as the method based on strain gauge measurements.

Further investigations are necessary for the evaluation of non-uniform pressure distributions along the wing span. In order to get better results for the fitting of the pressure curves, it is also necessary to increase the number of sensors in the front and rear sections of the airfoil. For new constructions of the wing glove, the number of sensors and their location should be improved. This will also improve the variation of the shear force results for different flight maneuvers. From a total of 122 flight maneuvers, only 48 maneuvers with almost steady load conditions were analyzed and evaluated in this paper. During the flight tests also unsteady maneuvers were performed like stalls and pull-up-push-over maneuvers. Future investigations will concentrate on the evaluation of these unsteady flight maneuvers. In that case, the measurement of unsteady flow characteristics and the associated dynamic load changes will be investigated in more detail, like e.g. the separation of flow during stall.

\section{Acknowledgments}

The authors would like to thank the company messWERK GmbH Braunschweig for support with data acquisition and flight test instrumentation equipment. We especially would like to thank the colleagues and members of the DLR Department for Flight Experiments, the DLR Flying Group Braunschweig and the Akaflieg Braunschweig for flight test support and towing of the glider aircraft.

\section{References}

[1] Skopinski, T. H., Aiken, J., William S, and Huston, W. B., "Calibration of strain-gage installations in aircraft structures for the measurement of flight loads," NACA Report 1178, 1954.

[2] Kottkamp, E., Wilhelm, H., and Kohl, D., “Strain Gauge Measurements on Aircraft,” AGARDograph, , No. $160,1976$. 
[3] Jenkins, J. M., Kuhl, A. E., and Carter, A. L., "Strain Gage Calibration of a Complex Wing," Journal of Aircraft, Vol. 14, No. 12, 1977, pp. 1192-1196. doi:10.2514/3.58914.

[4] Eckstrom, C. V., "Flight loads measurements obtained from calibrated strain-gage bridges mounted externally on the skin of a low-aspect-ratio wing," NASA TN D-8349, 1976.

[5] Lee, J.-R., Ryu, C.-Y., Koo, B.-Y., Kang, S.-G., Hong, C.-S., and Kim, C.-G., "In-flight health monitoring of a subscale wing using a fiber Bragg grating sensor system," Smart Materials and Structures, Vol. 12, No. 1, 2003 , pp. 147-155. doi:10.1088/0964-1726/12/1/317.

[6] López-Higuera, J., Cobo, L., Incera, A., and Cobo, A., "Fiber optic sensors in structural health monitoring," Journal of Lightwave Technology, Vol. 29, No. 4, 2011, pp. 587-608. doi:10.1109/JLT.2011.2106479.

[7] Yip, L., Vijgen, P., Hardin, J., and van Dam, C., "In-Flight pressure measurements on a subsonic transport high-lift wing section," Journal of Aircraft, Vol. 32, No. 3, 1995, pp. 529-538. doi:10.2514/3.46752.

[8] Reeh, A., Weismüller, M., and Tropea, C., "Free-flight investigation of transition under turbulent conditions on a laminar wing glove," 51st AIAA Aerospace Sciences Meeting including the New Horizons Forum and Aerospace Exposition $2013,2013$.

[9] Reeh, A., and Tropea, C., "Behaviour of a natural laminar flow aerofoil in flight through atmospheric turbulence," Journal of Fluid Mechanics, Vol. 767, 2015, pp. 394-429. doi:10.1017/jfm.2015.49.

[10] Greff, E., "In-flight measurement of static pressures and boundary-layer state with integrated sensors," Journal of Aircraft, Vol. 28, No. 5, 1991, pp. 289-299. doi:10.2514/3.46027, 5.

[11] Holland, M., Eccles, L., and Paradis, L., "A pressure belt system for an airborne pressure survey," SIcon 2001 - Proceedings of the 1st ISA/IEEE Sensors for Industry Conference, 2001, pp. 156-161. doi:10.1109/SFICON.2001.968521.

[12] Tanielian, M., "MEMS multi-sensor system for flight testing," Proceedings of SPIE - The International Society for Optical Engineering, Vol. 4559, 2001, pp. 120-129. doi:10.1117/12.443026.

[13] Tanielian, M., and Kim, N., "Pressure Belt: An Integrated Multisensor System," Proceedings of IEEE Sensors, Vol. 1, No. 2, 2002, pp. 1182-1187.

[14] Ostrower, J., "787 first flight is just the start for gruelling programme," Flight Global, 2009. URL https://www . flightglobal. com/news/articles/787-first-flight-is-just-the-start-for-gruelling-pro-336319/ last Accessed: 9th May 2019.

[15] Goold, I., "Full Circle - The Boeing 787's troubled service entry and new engine technology have given the Airbus A330 a new lease of life," Aerospace Testing International, March 2018, edited by B. Sampson, 2018.

[16] Viana, M. V. P., “Time-Domain System Identification of Rigid-Body Multipoint Loads Model," American Institute of Aeronautics and Astronautics (AIAA) - Atmospheric Flight Mechanics Conference (AFM) - AVIATION 2016, 2016.

[17] Viana, M. V. P., "Sensor calibration for calculation of loads on a flexible aircraft," 16th International Forum on Aeroelasticity and Structural Dynamics - IFASD 2015, 2015.

[18] Viana, M. V. P., "Sensor Calibration for Calculation of Loads on the DLR Discus-2c Sailplane," DLR Technical Report $111-2015 / 21,2015$.

[19] Datasheet BMP280 Digital Pressure Sensor, Bosch Sensortec GmbH, Jan. 2018. Rev. 1.19.

[20] Jategaonkar, R. V., Flight Vehicle System Identification, American Institute of Aeronautics and Astronautics, Reston, 2015. doi:10.2514/4.866852.

[21] Drela, M., "XFOIL: an analysis and design system for low Reynolds number airfoils." Low Reynolds number aerodynamics : proceedings of the conference, Notre Dame, Indiana, USA, 5-7 June 1989, edited by T. Mueller, Berlin, Germany, Springer-Verlag, 1989, pp. 1-12.

[22] McCormick, B. W., Aerodynamics, Aeronautics, and Flight Mechanics, John Wiley \& Sons, 1995. 